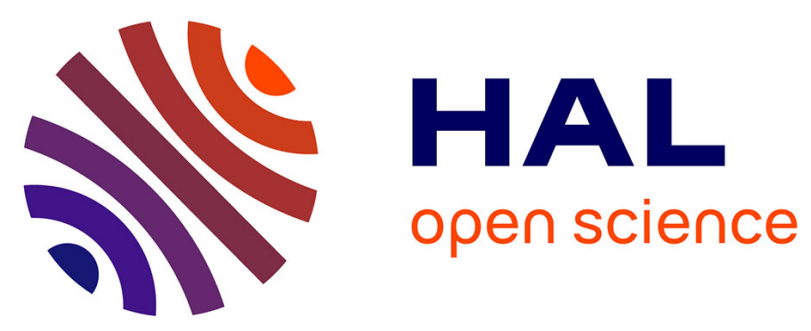

\title{
New substrates and interactors of the mycobacterial Serine/Threonine protein kinase PknG identified by a tailored interactomic approach
}

Magdalena Gil, Analía Lima, Bernardina Rivera, Jéssica Rossello, Estefanía Urdániz, Alessandro Cascioferro, Federico Carrión, Annemarie Wehenkel, Marco Bellinzoni, Carlos Batthyany, et al.

\section{To cite this version:}

Magdalena Gil, Analía Lima, Bernardina Rivera, Jéssica Rossello, Estefanía Urdániz, et al.. New substrates and interactors of the mycobacterial Serine/Threonine protein kinase PknG identified by a tailored interactomic approach. Journal of Proteomics, 2019, 192, pp.321-333. 10.1016/j.jprot.2018.09.013 . pasteur-02749223

\section{HAL Id: pasteur-02749223}

\section{https://hal-pasteur.archives-ouvertes.fr/pasteur-02749223}

Submitted on 16 Jun 2020

HAL is a multi-disciplinary open access archive for the deposit and dissemination of scientific research documents, whether they are published or not. The documents may come from teaching and research institutions in France or abroad, or from public or private research centers.
L'archive ouverte pluridisciplinaire HAL, est destinée au dépôt et à la diffusion de documents scientifiques de niveau recherche, publiés ou non, émanant des établissements d'enseignement et de recherche français ou étrangers, des laboratoires publics ou privés. 
1 New substrates and interactors of the mycobacterial Serine/Threonine protein kinase PknG

2 identified by a tailored interactomic approach

4 Magdalena Gil $^{\text {§ \# }}$, Analía Lima ${ }^{\mathrm{a} \#}$, Bernardina Rivera ${ }^{\mathrm{a}}$, Jessica Rossello ${ }^{\mathrm{a}}$, Estefanía Urdániz ${ }^{\mathrm{b}}$, Alessandro

5 Cascioferro $^{\mathrm{c}}$, Federico Carrión ${ }^{\mathrm{d}}$, Annemarie Wehenkel ${ }^{\mathrm{e}}$, Marco Bellinzoni ${ }^{\mathrm{e}}$, Carlos Batthyány ${ }^{\mathrm{a}}$, Otto

6 Pritsch $^{\mathrm{d}}$, Ana Denicola ${ }^{\mathrm{f}}$, María N. Alvarez ${ }^{\mathrm{g}}$, Paulo C. Carvalho ${ }^{\mathrm{h}}$, María-Natalia Lisa ${ }^{\mathrm{e}, \mathrm{i}}$, Roland Brosch ${ }^{\mathrm{c}}$,

7 Mariana Piuri ${ }^{\mathrm{b}}$, Pedro M. Alzari ${ }^{\mathrm{e}}$, Rosario Durán ${ }^{\mathrm{a}}$ *

8

9 a Unidad de Bioquímica y Proteómica Analíticas, Institut Pasteur de Montevideo \& Instituto de

10 Investigaciones Biológicas Clemente Estable, Uruguay.

$11{ }^{\mathrm{b}}$ Departamento de Química Biológica, Facultad de Ciencias Exactas y Naturales, Universidad de Buenos

12 Aires, Argentina.

$13{ }^{\mathrm{c}}$ Integrated Mycobacterial Pathogenomics Unit, Institut Pasteur, Paris, France.

14 d Unidad de Biofísica de Proteínas, Institut Pasteur de Montevideo, Uruguay.

15 e Unité de Microbiologie Structurale \& CNRS URA 2185, Institut Pasteur, Paris, France.

16 f Laboratorio de Fisicoquímica Biológica, Facultad de Ciencias, Universidad de la República, Uruguay.

17 g Departamento de Bioquímica, Facultad de Medicina, CEINBIO, Universidad de la República, Uruguay.

$18{ }^{\mathrm{h}}$ Laboratory for Proteomics and Protein Engineering, Carlos Chagas Institute, Fiocruz-Paraná, Brazil.

$20 \S$ Magdalena Gil's current address is Unit of Dynamics of Host-Pathogen Interactions, Institut Pasteur,

21 Paris, France.

22 i María-Natalia Lisa's current address is Instituto de Biología Molecular y Celular de Rosario (IBR,

23 CONICET-UNR), Ocampo y Esmeralda, S2002LRK, Rosario, Argentina.

$24{ }^{\#}$ These authors contributed equally to this work 
* Corresponding author: Rosario Durán, Unidad de Bioquímica y Proteómica Analíticas, Institut

27 Pasteur de Montevideo, Mataojo 2020, Montevideo 11400, Uruguay, Tel: +598 2 5220910, FAX: +598 2 5224185, e-mail: duran@pasteur.edu.uy

Keywords: PknG; Serine/Threonine protein kinase; glutamine synthetase; FhaA; Affinity purificationmass spectrometry; Mycobacterium tuberculosis.

Abbreviations: AP-MS, affinity purification-mass spectrometry; DIA, Differential In gel Analysis; FHA, forkhead associated domain; GS, glutamine synthetase; STD, internal standard; STPK, Serine/Threonine protein kinase; TPR, tetratricopeptide repeats; WT, wild type.

\section{Abstract}

PknG from Mycobacterium tuberculosis is a multidomain Serine/Threonine protein kinase that regulates bacterial metabolism as well as the pathogen's ability to survive inside the host by still uncertain mechanisms. To uncover PknG interactome we developed an affinity purification-mass spectrometry strategy to stepwise recover PknG substrates and interactors; and to identify those involving PknG autophosphorylated docking sites. We report a confident list of 7 new putative substrates and 66 direct or indirect partners indicating that PknG regulates many physiological processes, such as nitrogen and energy metabolism, cell wall synthesis and protein translation. GarA and the 50S ribosomal protein L13, two previously reported substrates of PknG, were recovered in our interactome. Comparative proteome analyses of wild type and $p k n G$ null mutant $M$. tuberculosis strains provided evidence that two kinase

47 interactors, the FHA-domain containing protein GarA and the enzyme glutamine synthetase, are indeed endogenous substrates of PknG, stressing the role of this kinase in the regulation of nitrogen metabolism. Interestingly, a second FHA protein was identified as a PknG substrate. Our results show that PknG

50 phosphorylates specific residues in both glutamine synthetase and FhaA in vitro, and suggest that these 51 proteins are phosphorylated by PknG in living mycobacteria. 


\section{Introduction}

Mycobacterium tuberculosis, the etiological agent of tuberculosis, is a major health problem and

the main cause of death due to a single infectious agent. According to the World Health Organization, this pathogen has caused 10.4 million new cases and 1.7 million deaths worldwide during 2016 [1]. One crucial feature of M. tuberculosis pathogenesis is its ability to respond to environmental signals switching from dormant to replicating bacilli in different disease stages $[2,3]$. In particular, signal transduction pathways involving protein phosphorylation play key roles in the adaptive response of $M$. tuberculosis $[4,5]$. mycobacteria changed dramatically in response to environmental stimuli [5-7]. However, the identification of the specific enzyme responsible for every phosphorylation event and the characterization

64 of the function of each phosphoprotein still lags behind $[8,9]$. proteins. These mycobacterial STPKs have been related to the regulation of several processes, including infection [11-14]. Among them, PknG became of special interest as it was found to play dual roles in mycobacterial metabolism and pathogenicity through mechanisms still not completely understood. On one hand, disruption of $p k n G$ gene reduced $M$. tuberculosis viability in vitro and in infection models, and caused retarded mortality in highly susceptible infected mice [12,15]. Furthermore, a M. bovis BCG pknG null mutant strain was found to be unable to block phagosome maturation in infected macrophages, and it has been proposed that PknG secretion and interference with the host cell signalling could be the underlying mechanism of this effect [16]. Very recently, other reports also support a role for PknG in 
environments $[17,18]$. In addition, the deletion of $p k n G$ caused a multidrug sensitive phenotype in contraposition to the intrinsic antibiotic resistance of pathogenic mycobacteria [19].

Other functions have been reported for PknG in bacterial metabolism. We have shown that PknG participates in the control of glutamate metabolism via the phosphorylation of the endogenous regulator GarA [20]. In addition, it was shown that PknG can regulate the activity of the Nudix hydrolase RenU through the phosphorylation of the ribosomal protein L13 [21]. Besides these better characterized substrates, very recent phosphoproteomics and protein microarray analysis have expanded the list of putative PknG substrates and interactors [22-24], and few candidates were further selected to test its interaction with, or phosphorylation by PknG. These studies suggest that RmlA and MurC activities are also regulated by PknG mediated phosphorylation $[22,24]$.

PknG presents a unique modular domain organization. Flanking the conserved catalytic kinase domain, $\mathrm{PknG}$ has a $\mathrm{N}$-terminal rubredoxin-like domain and a C-terminal domain composed of tetratricopeptide repeats (TPR). The Rbx domain of PknG has been the only protein motif found to regulate the intrinsic kinase activity $[25,26]$, while the TPR domain can lead to PknG dimerization without evident effects on the kinase activity [26,27]. Besides, in its N-terminal end PknG has a possibly unstructured extension with up to four autophosphorylation sites that act as essential anchoring points for the recruitment of the forkhead-associated (FHA) domain-containing regulator GarA [20]. FHA domains specifically recognize phosphorylated Thr residues and play important roles in phosphorylation dependent signal transduction [28]. Thus, PknG phosphorylates GarA within a conserved N-terminal motif, triggering the self-recognition of the phosphorylated residue by the FHA domain in the C-terminus of the molecule $[20,29,30]$. This interaction serves as a switch to activate/inhibit GarA control of downstream metabolic enzymes that use alpha-ketoglutarate as substrate [20,31].

The central role of PknG in mycobacterial physiology and virulence is well documented, but the molecular mechanisms underlying these effects, as well as the protein partners involved, are still poorly understood. Even when the list of putative substrates and interactors is rapidly expanding, there is very little overlap between these high-throughput studies and only few PknG substrates have been validated as 
104 physiologically relevant. This evidences that the identification of bona-fide kinase substrates and the

105 processes they regulate still represents a challenge from a methodological point of view and requires the 106 use of multiple experimental approaches.

107 With the aim of contributing to a better understanding of the biological processes regulated by PknG, 108 we developed a tailored interactomic approach. We combined the use of different constructions of PknG 109 with specific sequential elution steps to identify PknG mediated protein complexes and, in particular, to 110 discriminate those interactions relying on PknG's autophosphorylated docking sites. First,

111 phosphorylation conditions were used to elute PknG substrates that, similar to the model substrate GarA, 112 are released after phosphorylation. Second, a phosphatase treatment was employed to disrupt interactions 113 mediated by phosphoresidues, including PknG autophosphorylated sites in the case of the whole length 114 construction. A third and final elution step was performed to recover the remaining interacting partners.

115 Using this experimental approach, we recovered 66 direct or indirect PknG partners, including the two 116 previously reported substrates GarA and the 50S ribosomal protein L13. Further, two other proteins 117 identified in the interactome of PknG, the enzyme glutamine synthetase (GS) and the protein FhaA, were 118 validated as PknG substrates in vitro and evidence that both proteins are endogenous substrates of this 119 kinase is provided. Altogether, our results suggest that PknG regulates a wide range of cellular processes 120 including protein translation, nitrogen assimilation and cell wall biosynthesis.

\section{Materials and methods}

\subsection{Preparation of mycobacterial lysates}

(WT) and a $p k n \mathrm{G}$ null mutant strain $(\Delta p k n \mathrm{G})$ were grown in Middlebrook $7 \mathrm{H} 9$ supplemented with $0.05 \%$

127 Tween ${ }^{\circledR} 80$ containing ADC supplement (BD Biosciences) until early-logarithmic phase. Cells were

128 washed and then resuspended in minimum medium supplemented with $10 \mathrm{mM}$ asparagine and cultured

129 for 4-5 additional days. Mycobacterium smegmatis MC $^{2} 155$ was grown in Sauton's medium 
supplemented with $0.05 \%$ Tween $^{\circledR} 80$ until logarithmic phase. Mycobacterial cells were harvested and

131 resuspended in PBS (M. tuberculosis) or $25 \mathrm{mM}$ HEPES, $150 \mathrm{mM} \mathrm{NaCl}, 1 \%$ glycerol, $1 \mathrm{mM}$ EDTA, pH

1327.4 (protein interaction buffer for M. smegmatis) plus Complete EDTA-free Protease Inhibitor Cocktail

133 (Roche). An equal amount of acid-washed glass beads $(\leq 106 \mu \mathrm{m}$, Sigma) was added to the cell pellet and

134 lysis was achieved by vortexing at top speed for $10 \mathrm{~min}$. Cell debris and beads were removed by

135 centrifugation and protein quantification in the supernatant was performed by densitometry analysis in

136 gel. Protein extracts of each strain were prepared from three independent biological replicates.

\subsection{Affinity purification and sequential elution of PknG interactors}

139 Full-length PknG and $\mathrm{PknG}_{\Delta 73}$ were produced as described before [20] and immobilized on NHS-

140 Activated Sepharose 4 Fast Flow (GE Healthcare) following the supplier's instructions. In mock

141 experiments, a control resin prepared by blocking active groups with ethanolamine was used.

PknG kinase activity was confirmed by using autophosphorylation and GarA phosphorylation assays as previously described [20,25]. Briefly, for autophosphorylation assay, immobilized kinase was

144 incubated with $1 \mathrm{mM} \mathrm{MnCl} 2$ in $50 \mathrm{mM}$ HEPES, pH 7.4, for $30 \mathrm{~min}$ at $37^{\circ} \mathrm{C}$ with or without (control) 500

$145 \mu \mathrm{M}$ ATP. Proteins immobilized in the resins were further digested overnight with trypsin (sequence

146 grade, Promega) and the peptides recovered were analyzed by MALDI-TOF MS (4800 MALDI-

147 TOF/TOF Analyzer, Abi Sciex) using linear mode and $\mathrm{m} / z$ range from 2000 to 6000. Phosphorylation of

148 the recombinant substrate was performed by incubation of immobilized PknG with GarA (final

149 concentration $70 \mu \mathrm{M}$ ) under the same conditions used for autophosphorylation assay, with or without

150 ATP. GarA phosphorylation status was verified by whole molecular mass measurements using a MALDI-

151 TOF mass spectrometer (4800 MALDI-TOF/TOF Analyzer, Abi Sciex) operated in linear mode.

152 Immobilized PknG, $\mathrm{PknG}_{\Delta 73}$ or control resin pre-equilibrated in the interaction buffer were incubated

153 with $800 \mu \mathrm{g}$ of $M$. smegmatis protein extract (three biological replicates) supplemented with $2 \mathrm{mM}$

154 EDTA, overnight at $4{ }^{\circ} \mathrm{C}$, with gentle agitation. The matrix was packed into Pierce ${ }^{\mathrm{TM}}$ Micro-Spin 
Columns (Thermo Scientific) and the retained proteins were eluted using the following protocol: (a) for the elution of interactors under phosphorylation conditions (E1), the $\mathrm{PknG}, \mathrm{PknG}_{\Delta 73}$ and control matrices were incubated with $1 \mathrm{mM} \mathrm{MnCl}_{2}$ and $500 \mu \mathrm{M}$ ATP in $50 \mathrm{mM}$ HEPES, pH 7.0, for $30 \mathrm{~min}$ at $37{ }^{\circ} \mathrm{C}$ and the eluted proteins were recovered; (b) for the elution of proteins under dephosphorylation conditions (E2), the resins were further incubated with $0.18 \mathrm{U} / \mu \mathrm{L}$ of calf intestine alkaline phosphatase (Roche) for $40 \mathrm{~min}$ at $37^{\circ} \mathrm{C}$ and the eluted proteins were recuperated; (c) for the elution of proteins under unspecific conditions (E3), the matrices containing the remaining interactors were incubated for $10 \mathrm{~min}$ at $25{ }^{\circ} \mathrm{C}$ with $70 \%$ ACN, $0.1 \%$ formic acid and the eluted proteins were collected. The whole experiment was run in triplicates, using independent biological samples, and each eluate was analyzed by nano-LC MS/MS.

\subsection{Production of recombinant FhaA}

Plasmid pLAM12-fhaA, for Strep-tag ${ }^{\circledR}$ II-FhaA overexpression in mycobacteria, was constructed by PCR amplification of Rv0020c (fhaA) from genomic DNA of M. tuberculosis H37Rv using the oligonucleotides

(FhaA-Fw) and ATATTGAATTCTCAGTGCATGCGGACGATGATC-3' (FhaA Rv), and cloning the insert

171 between the sites NdeI and EcoRI (underlined) in the extrachromosomally-replicating parent vector 172 pLAM12 [32], under the control of the M. smegmatis acetamidase promoter. FhaA-Fw contains a 5' 173 extension coding for the STAG II sequence (bold). Plasmid pLAM12-fhaA was used to transform 174 electrocompetent $M$. smegmatis $\mathrm{MC}^{2} 155$ cells. Transformants were grown in Middlebrook $7 \mathrm{H} 9$ broth 175 supplemented with ADC, $0.05 \%$ Tween ${ }^{\circledR} 80$ and kanamycine, and the expression of Strep-tag ${ }^{\circledR}$ II-FhaA 176 was induced by the addition of $0.2 \%$ acetamide during exponential growth. Strep-tag ${ }^{\circledR}$ II-FhaA (herein 177 after named FhaA) was purified using Strep-Tactin ${ }^{\circledR}$ Sepharose $^{\circledR}$ (IBA) and eluted in a competitive 178 manner with D-desthiobiotin. 

plasmid pCRT7::Rv2220 and cells were grown in autoinduction medium for $4 \mathrm{~h}$ at $37{ }^{\circ} \mathrm{C}$ and later overnight at $20^{\circ} \mathrm{C}$. GS was first purified by metal-affinity chromatography on a His-Trap FF crude column equilibrated in $50 \mathrm{mM}$ HEPES $\mathrm{pH} 8.0,500 \mathrm{mM} \mathrm{NaCl}, 5 \%$ glycerol and $5 \mathrm{mM}$ imidazol, using a linear imidazole gradient from 5 to $500 \mathrm{mM}$. Size exclusion chromatography was performed on fractions containing GS, using a Sephacryl S400 16/60 column (GE Healthcare) equilibrated in 20 mM HEPES pH 7.7, $150 \mathrm{mM} \mathrm{NaCl}, 3 \%$ glycerol and $1 \mathrm{mM} \mathrm{MgCl}_{2}$. Oligomeric state of GS was determined using either

189 Dynamic Light Scattering or native gels (NativePAGETM 3-12\% Bis-Tris Protein Gels, Thermo). Native 190 Mark Unstained Molecular Weight Marker (Thermo) was used as standard.

\subsection{Mapping FhaA or GS phosphorylation sites}

To assay FhaA as a substrate of PknG in vitro, a cell lysate of $M$. smegmatis MC $^{2} 155$ overexpressing 194 FhaA was initially loaded onto a Strep-Tactin ${ }^{\circledR}$ Sepharose ${ }^{\circledR}$ column (IBA). Immobilized FhaA was later 195 dephosphorylated with $0.07 \mathrm{U} / \mu \mathrm{L}$ calf intestine alkaline phosphatase (Roche) for $40 \mathrm{~min}$ at $37^{\circ} \mathrm{C}$. Dephosphorylated FhaA was then eluted and further incubated with $1 \mathrm{mM} \mathrm{MnCl}, 250 \mu \mathrm{M}$ ATP and 10 nM PknG, for $30 \mathrm{~min}$ at $37^{\circ} \mathrm{C}$. As a control, we performed the same experiment in the absence of PknG. Additionally, to identify the sites of FhaA phosphorylated in vivo, FhaA was purified from M. smegmatis cells transformed with plasmid pLAM12-fhaA and induced with acetamide. $\mu \mathrm{M}$ ATP and PknG (molar ratio PknG:GS 1:150), for 30 min at $37{ }^{\circ} \mathrm{C}$. 
For surface plasmon resonance analysis, FhaA was diluted in $10 \mathrm{mM}$ sodium acetate $\mathrm{pH} 4.5$ at a concentration of $5 \mu \mathrm{g} / \mathrm{mL}$ and immobilized on a CM5 sensorchip by standard amine coupling. A BIACORE 3000 (Biacore AB, Uppsala, Sweden) was used, achieving a final density of 25 RU. Phosphorylated PknG and $\mathrm{PknG}_{\Delta 73}$ were diluted in $10 \mathrm{mM}$ HEPES pH 7.4, 0.15 M NaCl, 3 mM EDTA, $210 \quad 0.005 \% \mathrm{v} / \mathrm{v}$ Surfactant P20 to a final concentration of $30 \mathrm{nM}$ and injected during 3 min at a flow rate of $21180 \mu \mathrm{L} / \mathrm{min}$ over the immobilized and a reference surfaces. Regeneration was achieved by extensively 212 washing with running buffer. All injections were done at $25^{\circ} \mathrm{C}$ and double referenced by subtracting the 213 reference cell signal and a buffer injection.

\subsection{Differential Gel Electrophoresis (DIGE)}

216 Comparative proteome analyses between wild type $M$. tuberculosis and $\Delta p k n G$ strains were performed

217 on three biological replicates, using the Ettan DIGE System (GE Healthcare) and following the 218 manufacturer's instructions. Samples were purified using the 2D Clean-up kit (GE Healthcare) and 219 protein pellets were solubilized in $30 \mathrm{mM}$ Tris $\mathrm{pH}$ 8.5, $7 \mathrm{M}$ urea, $2 \mathrm{M}$ thiourea, 4\% CHAPS. multiplex matching of DIGE images, spot normalization and calculation of spots abundance changes.

222 Then, the STD and the protein samples WT and $\Delta p k n G(50 \mu \mathrm{g}$ each) were differentially labelled with the 223 N-hydroxysuccinimidyl ester derivatives of the cyanine dyes Cy2, Cy3, and Cy5 following the 224 manufacturer's instructions for minimal labelling (GE Healthcare, Munich, Germany). The differentially 225 labelled WT and $\Delta p k n G$ samples together with the STD were mixed and rehydration solution (7 M urea, 2 226 M thiourea, 4\% CHAPS, 0.5\% IPG Buffer 4-7 (GE Healthcare)) was added. This mixture was later used 227 to rehydrate IPG strips (13 cm pH 4-7) overnight. Isoelectric focusing was performed in an IPGphor Unit 228 (Pharmacia Biotech) applying the following voltage profile: constant phase of $500 \mathrm{~V}$ for $1 \mathrm{~h}$, linear 229 increase from to $5000 \mathrm{~V}$ in $1 \mathrm{~h}$, followed by another linear increase to $8000 \mathrm{~V}$ in $2 \mathrm{~h} 30 \mathrm{~min}$, and a final 230 constant phase of $8000 \mathrm{~V}$ in $35 \mathrm{~min}$, reaching a total of $17.5 \mathrm{kVh}$. Prior to running the second dimension, 

performed on $12.5 \%$ SDS-PAGE, at $20{ }^{\circ} \mathrm{C}$, in a SE 600 Ruby Standard Dual Cooled Vertical Unit (GE Healthcare). Gels were scanned with a Typhoon FLA 9500 laser scanner (GE Healthcare), at a resolution of $100 \mu \mathrm{m}$, using laser wavelengths and filters recommended for each dye. Differential In-gel Analysis (DIA) was used for spots co-detection, spot quantification by normalization and calculation of the ratio between different samples in the same gel. Biological Variation Analysis

240 software module was used to perform inter-gel matching and statistical analyses. An unpaired Student's t-

241 test was employed to determine if the standardized spot volume abundances from the triplicate samples

242 showed significant changes between the two conditions. Spots displaying a pI shift profile consistent with

243 the presence of post-translational modification such as phosphorylation (spots in trains with increased 244 abundance in WT strain), significant volume abundances differences $(\mathrm{p} \leq 0.05)$ and a fold change of at 245 least $25 \%$ were filtered and selected for further analyses.

246 To confirm the phosphorylation status of the differential spots, we compared wild type M. tuberculosis 247 protein extracts pre-labelled with CyDyes with and without treatment with alkaline phosphatase (Cy3 and Cy2 respectively), using DIA analysis (DeCyder ${ }^{\mathrm{TM}}$ 2D software). The same strategy was use to compare $\Delta p k n G$ protein extracts with and without previous incubation with recombinant $\mathrm{PknG}$ under

250 phosphorylation conditions (Cy5 and Cy2 respectively).

\subsection{Sample preparation for MS analysis}

253 Protein mixtures obtained from affinity purification (AP) experiments were reduced and alkylated with 254 iodoacetamide prior to treatment with trypsin. Peptide quantification was performed at $280 \mathrm{~nm}$ using a 255 NanoDrop 1000 spectrophotometer (Thermo Scientific) prior to its analysis by nano-LC MS/MS (LTQ 256 Velos, Thermo). 

MALDI-TOF MS. Briefly, spots were in-gel digested with trypsin and the resultant peptides were extracted using aqueous $60 \% \mathrm{ACN} / 0.1 \%$ trifluoroacetic acid, and concentrated by vacuum drying.

261 Samples were desalted using C18 micro-columns ( $\mathrm{C}_{18}$ OMIX pipette tips, Agilent) and eluted directly

262 onto the sample plate for MALDI-MS with $\alpha$-cyano-4-hydroxycinnamic acid matrix solution in aqueous $60 \% \mathrm{ACN} / 0.1 \%$ trifluoroacetic acid. Additionally, some spots were analyzed by nano-LC MS/MS, in this 264 case elution was performed in 70\% ACN/0.1\% formic acid.

\subsection{Protein identification by MALDI-TOF/TOF MS}

267 Tryptic peptides obtained from the spots of the silver stained master gel were analysed using a 4800

268 MALDI-TOF/TOF Analyzer (Abi Sciex, Framingham, MA) equipped with 355mn Nd:YAG laser.

269 Spectra were acquired in reflector mode using the following parameters: detector voltage: $1.75 \mathrm{kV}$; laser ranging from 50 and $70 \%$ of maximum intensity; 160 shots per subspectra and 25 subspectra acquired.

271 MS/MS analyses of selected peptides were performed for all the analyzed spots in DIGE experiments. For

272 MS/MS spectra acquisition source 1 and source 2 voltages were $8 \mathrm{kV}$ and $15 \mathrm{kV}$, respectively.

Proteins were identified by database searching of measured $\mathrm{m} / \mathrm{z}$ values using the MASCOT search engine (Matrix Science http://www.matrixscience.com) in the sequence Query mode, using the database from NCBI (20160821) and restricting the taxonomy to the Mycobacterium tuberculosis complex. The

276 following search parameters were set: monoisotopic mass tolerance, $0.03 \mathrm{Da}$; fragment mass tolerance,

$2770.5 \mathrm{Da}$; methionine oxidation and phosphorylation of Ser, Thr and Tyr as variable modification, cysteine

278 carbamidomethylation as fixed modification and one missed tryptic cleavage allowed. Significant protein

279 scores $(\mathrm{p}<0.05)$, at least three peptides per protein and at least one fragmented peptide with ion

280 significant score $(\mathrm{p}<0.05)$ per protein were used as criteria for positive identification. 
Samples from AP experiments, selected DIGE spots and phosphorylated proteins were analyzed by nano-LC MS/MS. Each sample was injected into a nano-HPLC system (EASY-nLC 1000, Thermo Scientific) fitted with a reverse-phase column (EASY-Spray column, $50 \mathrm{~cm} \times 75 \mu \mathrm{m}$ ID, PepMap RSLC C18, $2 \mu \mathrm{m}$, Thermo Scientific). For AP experiments, peptides were separated on a linear gradient of solvent $\mathrm{B}(\mathrm{ACN} 0.1 \%$ formic acid (v/v)) from $5 \%$ to $55 \%$ in $75 \mathrm{~min}$. In the case of DIGE and DIA spots, as well as analysis of phosphorylation status of recombinant proteins, the gradient was modified as follow: linear gradient of solvent B from 5\% to 55\% in $60 \mathrm{~min}$. In both cases, the flow rate was set at 250 $\mathrm{nL} / \mathrm{min}$ at $45^{\circ} \mathrm{C}$.

Peptide analysis was carried out in a LTQ Velos nano-ESI linear ion trap instrument (Thermo Scientific) set in a data-dependent acquisition mode using a dynamic exclusion list. NSI-source parameters were set as follows: spray voltage $(\mathrm{kV}): 2.3$ and $260{ }^{\circ} \mathrm{C}$ capillary temperature. Mass analysis was performed with Xcalibur 2.1 in two steps: (1) acquisition of full MS scan in the positive ion mode with $\mathrm{m} / z$ between 400 and $1200 \mathrm{Da}$, (2) CID fragmentation of the ten most intense ions with the following parameters; normalized collision energy: 35, activation Q: 0.25; activation time: $15 \mathrm{~ms}$.

\subsection{Proteomic data analysis}

A target-decoy database including sequences from $M$. smegmatis strain ATCC 700084/MC ${ }^{2} 155, M$. tuberculosis strain ATCC 25618/H37Rv downloaded from Uniprot consortium in November 2014, and 127 most common mass spectrometry contaminants was generated using PatternLab for Proteomics (version 3.2.0.3) [35,36], giving rise a database with 10.712 entries. For analyses of AP samples and protein identification of selected spots from DIGE gel, the Comet search engine was set as follows: tryptic peptides; oxidation of methionine and phosphorylation of Ser, Thr or Tyr as variable modifications and carbamidomethylation as fixed modification; and $700 \mathrm{ppm}$ of tolerance from the measured precursor $\mathrm{m} / \mathrm{z}$. XCorr and Z-Score were used as the primary and secondary search engine scores, respectively.

307 Peptide spectrum matches were filtered using the Search Engine Processor (SEPro) using the following 308 parameters; acceptable FDR: $1 \%$ at the protein level; a minimum of two peptides per protein. All reported 
proteins have at least one unique peptide. Comparisons between proteins present in each elution of PknG,

$310 \mathrm{PknG}_{\Delta 73}$ and mock experiments was performed using PatternLab's Approximately Area Proportional

311 Venn Diagram module, and verification of statistical validity of the proteins was performed according to

312 the Bayesian model integrated into PatternLab for proteomics [36,37]. Briefly, the model considered

313 quantitative data and number of appearances in different biological replicates to assign p-values and

314 ultimately shortlist proteins that are likely to be real interactors.

315 For phosphosite identification of FhaA and GS recombinant proteins, Proteome Discoverer software

316 package (v.1.3.0.339, Thermo) with Sequest as search engine was used. Database was compiled from $M$.

317 tuberculosis strain ATCC 25618/H37Rv (April 2015) and M. smegmatis strain ATCC 700084/MC 155

318 (November 2014) Uniprot protein sequences. The precursor mass tolerance and fragment mass tolerance

319 were set as 1 Da and 1.5 Da respectively, oxidation of methionine and phosphorylation of Ser, Thr or Tyr

320 were set as variable modifications. Peptide validator node was used to perform a decoy database search,

321 and the target FDR (strict) was set to 0.01. Only high-confident peptides with strict target false discovery

322 rate were considered. To localize phosphosites in validated sequences phosphoRS node was used. In

323 addition, we considered positive phosphosite identification when more than one spectrum for the

324 phosphopeptide was obtained. Manual inspection of the MS/MS spectra was performed to corroborate

325 peptide and phosphorylation site assignments.

\subsection{Bioinformatic analyses}

328 The list of M. smegmatis identified proteins was converted to M. tuberculosis orthologs and classified 329 using TubercuList (M. tuberculosis H37Rv database, March 2013 release 27 [38]). If a protein ortholog 330 was absent in TubercuList, a blastp sequence and similarity search was performed using M. smegmatis 331 sequence as query and $M$. tuberculosis complex (taxid: 77643) as database and a statistical significance 332 threshold of 10 (default value) [39]. Protein-protein interactions analyses were performed with STRING 333 database (v 10.0) (http://string-db.org/), using the entire set of putative PknG's interactors (MSMEG 334 identifiers) as an input gene list, and the following parameters: "known interactions" (from curated 
databases and experimentally determined) and a minimum interaction score of 0.7 (high confidence) [40].

336 A statistical overrepresentation test of GO biological processes (release 20160715) was performed using

337 the Panther Server (http://pantherdb.org) version 11.1. The release date of GO ontology dataset was 2017-

338 01-26. Mycobacterium tuberculosis database as reference list and the Tuberculist gene identifiers were 339 used [41].

\subsection{Data availability}

342 The mass spectrometry proteomics data have been deposited to the ProteomeXchange Consortium via the 343 PRIDE partner repository with the dataset identifier PXD005950 [42]. Reviewer account details to access 344 to proteomics data: Username: reviewer87925@ebi.ac.uk; Password: whLcyv5a.

\section{Results}

\subsection{An affinity chromatography-sequential elution strategy to find new PknG protein interactors}

To identify PknG protein partners we developed an affinity chromatography-sequential elution strategy to stepwise recover PknG interactors with different binding modes and to specifically discriminate those recruited through PknG autophosphorylated docking sites. PknG was initially immobilized on NHS-Activated Sepharose and incubated with ATP/Mn ${ }^{2+}$ to allow autophosphorylation,

352 which was later verified by MALDI-TOF MS analysis of tryptic digestion mixtures. As shown in 353 Supplementary Figure 1A, after the autophosphorylation reaction we observed an intense signal 354 corresponding to the diphosphorylated ion of sequence 10-60 $(\mathrm{m} / \mathrm{z}, 5556.3)$, in agreement with previous 355 results [20]. We also verified that the immobilized kinase behaved as an active enzyme towards the 356 substrate GarA (Supplementary Figure 1B). In parallel, we immobilized an active deletion mutant of 357 PknG lacking the phosphorylatable sequence 1-73 $\left(\mathrm{PknG}_{\Delta 73}\right)$ that participates in the recruitment of the 358 FHA-containing substrate GarA [20]. Thus, immobilized $\mathrm{PknG}, \mathrm{PknG}_{\Delta 73}$ and control resins were used to 359 fish out interacting proteins from total M. smegmatis protein extracts. First, elution was performed under 360 phosphorylation conditions $\left(\mathrm{ATP} / \mathrm{Mn}^{2+}\right)$ to recover substrates released after its phosphorylation by the 
immobilized kinase (E1). A subsequent elution step was performed under dephosphorylation conditions, sites in the case of the whole length PknG. Finally, the remaining interacting partners were recovered in a third elution step (E3). For each elution step, positive interactors were identified by comparison with mock experiments, using quantitative data and number of appearances in different biological replicates to assign $\mathrm{p}$-values (filtering options, $\mathrm{p}<0.05$ ) using Patternlab for Proteomics software.

\subsection{Identification of new $P k n G$ substrates and protein interactors}

Eight proteins were identified in at least two of three biological replicates of E1 when using full-length PknG as bait, whereas six of them were also recovered with $\mathrm{PknG}_{\Delta 73}$ (Tables 1 and S1). Interestingly, we could not identify proteins in any of the three replicates of mock resin. Therefore, the kinase N-terminal segment was critical for the recruitment of two putative phosphorylatable interactors. As expected, one of these proteins is the regulator GarA, validating our experimental approach. The other protein in this group was the enzyme glutamine synthetase 1 (GS, MSMEG_4290). While GarA contains an FHA domain that specifically recognizes pThr residues in PknG, no phospho-recognition domain has been predicted for GS, and its possible interaction through the N-terminal extension of PknG deserves further investigation. No protein was identified exclusively in the interactome of $\mathrm{PknG}_{\Delta 73}$ under the same conditions. However,

378 six proteins were systematically recovered using both full-length $\mathrm{PknG}$ and $\mathrm{PknG}_{\Delta 73}$, namely chaperone

379 protein DnaK, alcohol dehydrogenase, $60 \mathrm{kDa}$ chaperonin 1, 4Fe-4S ferredoxin, iron-sulfur binding 380 protein, inorganic pyrophosphatase and acetyl-/propionyl-coenzyme A carboxylase alpha chain.

381 Interestingly, six out of the eight proteins recovered in E1 have been previously reported as 382 phosphorylated proteins (Table S2).

383 Under dephosphorylation conditions (E2), the FHA protein FhaA (MSMEG_0035) was the only 384 protein systematically recovered when using full-length $\mathrm{PknG}$ as bait, but not $\mathrm{PknG}_{\Delta 73}$ or the mock resin 385 (Tables 1 and S3). The presence of a pThr recognition domain in FhaA possibly explains its specific 
interaction with the $\mathrm{N}$-terminal segment of the kinase. Additionally, 45 proteins were statistically significantly identified as interactors in E2 when compared to mock experiments using both baits, including PknG itself (Table S3). Among these proteins we recovered the previously reported substrate of PknG, the 50S ribosomal protein L13 [21], highlighting the usefulness of our interactomic approach to identify bona-fide kinase substrates. GS was also detected in all replicates of E2 elution with very high numbers of spectra assigned to its sequence, strongly supporting that this protein is indeed a PknG interactor. The fact that GS was recovered using both PknG constructs suggests that, besides the Nterminal segment of PknG, the kinase core and/or the TPR domain also play a role in its direct or indirect binding. Notably, 23 out of 46 proteins recovered in E2 are ribosomal proteins, including 7 known interactors of the ribosomal protein L13 (RplR, RplF, RplX, RplV, RplC, RplB and RplA), reflecting the recovery of physiologically relevant protein complexes (https://string-db.org/).

In the third elution step (E3) we employed astringent conditions to recover the remaining interactors of

398 PknG. Using the statistical model of Venn Diagram analysis for comparison with mock experiments, 24 399 proteins were identified in this fraction using whole length PknG and 22 of them were also recovered 400 using $\mathrm{PknG}_{\Delta 73}$, evidencing a confident list of partners (Table S4).

401 Overall, we identify 66 proteins in the PknG interactome (Table S5). Bioinformatics analysis showed 402 that our set of putative PknG partners presented a significant enrichment of previously reported proteinprotein interactions (PPI enrichment p-value $<1.0 \mathrm{e}-16)$. The biological processes that were statistically enriched in the PknG interactome were: ATP synthesis coupled proton transport; proton transmembrane transport; translation and ribosome biogenesis (Table S6). Moreover, the differential analysis of the interactome of $\mathrm{PknG}$ and $\mathrm{PknG}_{\Delta 73}$ indicates that GarA and FhaA are recruited exclusively by full-length

407 PknG, pointing to a specific interaction with pThr residues of the kinase N-terminal extension.

\subsection{Comparative analysis of the proteomes of wild type M. tuberculosis and a PknG-null mutant}

410 To evaluate if the newly identified interactors could represent physiological substrates of PknG, we 411 compared the proteomes of wild type $M$. tuberculosis and a $p k n G$ deletion mutant $(\Delta p k n G)$ by difference 
412 in gel electrophoresis (DIGE). This approach allows an estimation of the relative abundance of

413 proteoforms with different isoelectric points $(\mathrm{pI})$, representing therefore a valuable tool for the analysis of

414 kinase substrates. Image analysis led to the detection of around 2100 spots in each gel. Spots exhibiting

415 normalized abundance fold changes greater than $25 \%$ and p-values lower than 0.05 (Student's t-test) were

416 considered as differential spots. Not surprisingly, the differential spots that presented the largest overall

417 fold changes could be assigned by LC-MS/MS analysis to GarA (Fig. 1A and B). Four spots

418 corresponding to GarA proteoforms with different apparent pI values (spots 1 to 4) presented decreased

419 normalized abundance in the $\Delta p k n G$ strain, with fold changes ranging from 1.52 to 4.38 (Fig. 1B and

420 Table S7). At the same time, the more basic GarA spot (spot 5 in Figure 1) was overrepresented in

$421 \Delta p k n G$ strain, with an increased normalized abundance of 2.30 (Fig. 1B and Table S7). The pattern of

422 phosphorylated proteoforms of GarA was previously reported [30], and our results are consistent with

423 diminished levels of GarA phosphorylation in the $\Delta p k n G$ strain. In fact, $\mathrm{Thr}_{21}$, the specific residue

424 modified by PknG, was unequivocally detected as phosphorylated in the most acidic proteoform of GarA

425 overrepresented in wild type M. tuberculosis (Table S7). This finding confirms previous reports

426 indicating that GarA is a physiological substrate of PknG [15,20] and demonstrates the utility of the

427 DIGE approach to identify protein substrates of this kinase.

428 The analysis by DIGE allowed the identification of an additional area with a differential pattern of

429 spots between wild type M. tuberculosis and the $\Delta p k n G$ mutant strain, possibly reflecting differences in

430 protein phosphorylation. Interestingly, these spots were identified as GS (Rv2220) (spots 6 to 13 in Fig.

431 1B and Table S7). However, we failed to detect phosphopeptides in these very faint spots by MS,

432 possibly due to a combination of the low amount of available protein and the analytical difficulties

433 associated with the ionization and fragmentation of the phosphorylated peptides. To evaluate if

434 phosphorylation by PknG could be responsible for these differential spots, we performed two

435 complementary experiments. First, we treated protein extracts from wild type M. tuberculosis with

436 alkaline phosphatase and analyzed changes in the relative abundance of spots by differential in gel 
analysis (DIA). Then, the same approach was used to compare protein extracts from the M. tuberculosis $\Delta p k n G$ mutant strain before and after incubation with PknG. Spots 6-8 were not completely resolved in this DIA gel, therefore they were analyzed as a single spot. Interestingly, the relative intensity of GS

440 acidic spots, that were differential between strains, decreased after the treatment with phosphatase (fold 441 change of 6.5 for spot 6-8 and fold change of 5.3 for GS spot 9) (Fig. 2). Additionally, the relative 442 intensity of GS spot 9, underrepresented in $\Delta p k n G$ strain, increased upon incubation with PknG (fold 443 change of 2.16), while spot 6-8 didn't show difference in its abundance.

However, incubations with PknG did not change the relative abundance of the more basic GS spots (spots 10-13). Overall, these results confirm that GS is modified by phosphorylation in vivo and show that the incubation of protein extracts of $M$. tuberculosis $\Delta p k n G$ with PknG can partially recapitulate the pattern of GS spots observed in the wild type strain.

\subsection{PknG phosphorylates specific residues of GS}

The results described above point to GS as a new PknG substrate. To further validate these results, and to identify the involved phosphoresidues, recombinant GS was incubated with PknG under

452 phosphorylation conditions using different enzyme:substrate ratios. Phosphorylated peptides were 453 subsequently identified by tryptic digestion and LC-MS/MS. After incubation with PknG, the N-terminal 454 region of GS was systematically phosphorylated, even with an enzyme:substrate molar ratio as low as 455 1:150. Two peptides of $m / z 833.4$ and 1168.2, corresponding to doubly charged ions of phosphorylated sequences 45-59 and 60-79 respectively, were detected. $\mathrm{Thr}_{77}$ were unequivocally identified as the phosphorylated residue within sequence 60-79 by phosphoRS analysis (pRS probability 100\%) (Fig. 3B and Table S8). In addition, manual inspection of the spectra indicated the presence of several y ions $\left(\mathrm{y}_{5}{ }^{+}\right.$, $\left.\mathrm{y}_{5}^{+}-\mathrm{H}_{3} \mathrm{PO}_{4}, \mathrm{y}_{7}{ }^{+}, \mathrm{y}_{7}{ }^{+}-\mathrm{H}_{3} \mathrm{PO}_{4}, \mathrm{y}_{8}{ }^{+}, \mathrm{y}_{8}{ }^{+}-\mathrm{H}_{3} \mathrm{PO}_{4}\right)$ that supports the phosphosite assignment. In the case of 460 sequence 45-59, $\operatorname{Ser}_{57}$ was identified as the most probably phosphorylated residue by phosphoRS (pRS 461 probability $\mathrm{S}_{57}: 98.9 \%$; $\operatorname{Ser}_{56:} 0.2 \%$ ), and this was confirmed by the presence of a signal corresponding to

$462 \mathrm{y}_{3}{ }^{+}$ion in MS/MS spectra (Fig. 3A and Table S8). While $\mathrm{Thr}_{77}$ is exposed to the solvent and relatively 
distant from the active site, $\operatorname{Ser}_{57}$ is located in close proximity to an oligomerization interface and points to the active site of an adjacent monomer in the dodecameric structure of GS (Fig. 4). Thus, it is tempting to speculate that phosphorylation of $\operatorname{Ser}_{57}$ could interfere with substrate binding and/or GS oligomerization. Additionally, a third phosphopeptide (residues 404-429) was systematically identified in phosphorylation assays (triply charged ion of $\mathrm{m} / \mathrm{z}$ 933.1), and although unambiguous phosphosite identification is not possible from the spectra obtained, $\mathrm{Thr}_{421}$ is the most probably phosphorylated residue within this sequence (pRS site probability 90.5\%) (Fig. 3C and Table S8). Further support of $\mathrm{Thr}_{421}$ phosphorylation by $\mathrm{PknG}$ is provided by the detection of sequence 404-429 with two modifications: phosphorylation $\left(\mathrm{Thr}_{421}\right)$ and AMPylation $\left(\mathrm{Tyr}_{406}\right)$. In this case, the presence in the MS/MS spectrum of the fragment ions $\mathrm{y}_{10}{ }^{+}, \mathrm{y}_{10}{ }^{+}-\mathrm{H}_{3} \mathrm{PO}_{4}$; and a small signal corresponding to $\mathrm{y}_{9}{ }^{+}-\mathrm{H}_{3} \mathrm{PO}_{4}$ point to $\mathrm{Thr}_{421}$ and not $\mathrm{Thr}_{420}$ as the phosphorylated residue (Fig. 3D and Table S8). Interestingly, $\mathrm{Thr}_{421}$ is close in the primary and the tertiary structure of the protein to the conserved residue $\mathrm{Tyr}_{406}$, whose AMPylation is a well-known mechanism for the modulation of bacterial GS activity $[43,44]$. The fact that the phosphorylation of this sequence can occur alone or in combination with the AMPylation of $\mathrm{Tyr}_{406}$ suggests that both residues could have related regulatory roles.

\subsection{FhaA as a new substrate of M. tuberculosis PknG}

M. tuberculosis encodes several FHA-containing proteins and all of them have been identified as substrates of multiple STPKs in vitro [30,45-48]. With our interactomic approach we systematically recovered one of these proteins, FhaA, as the only $\mathrm{PknG}$ interactor recovered under dephosphorylation conditions when using the full-length kinase but not $\mathrm{PknG}_{\Delta 73}$, suggesting that the interaction between FhaA and PknG strongly relies on the N-terminal pThr residues of PknG. A recent work reported the interaction of FhaA with several mycobacterial kinases, but failed to detect an interaction with PknG [24].

We employed surface plasmon resonance to determine whether FhaA is able to establish a direct interaction with PknG. Phosphorylated PknG formed a stable complex with immobilized FhaA whereas truncated $\mathrm{PknG}_{\Delta 73}$ lacking all phosphosites did not (Supplementary Fig. 2). Overall, our results show 
that PknG and FhaA can interact in the absence of additional factors and that the phosphorylated Nterminal extension of PknG is essential for this binding.

Then, we decided to assess FhaA phosphorylation by PknG in vitro. While no phosphopeptides were identified in LC-MS/MS analysis of recombinant phosphatase-treated FhaA, two FhaA phosphopeptides were confidently identified after incubation of the protein with PknG, and the phosphorylated residues were unequivocally localized. A peptide of $m / z 472.3$ (corresponding to the triply charged ion of phosphorylated sequence 14-25) and a peptide of $\mathrm{m} / \mathrm{z} 816.2$ (corresponding to the doubly charged ion of phosphorylated sequence 108-120) were systematically detected in the phosphorylation assays. These two sequences mapped to the N-terminal domain of FhaA and MS/MS spectra allowed the unequivocal identification of $\mathrm{Thr}_{18}$ and $\mathrm{Thr}_{116}$ (pRs scores of 79 and 110 respectively; 100\% pRS probability in both cases) as the phosphorylated residues (Fig. 5A and Fig. 5B, respectively; and Table S8). Manual inspection of these spectra allowed the detection of numerous signals that support the specific

501 modification of these residues (Fig. 5A and $\mathbf{B}$, respectively).

502 To determine if these sites were also phosphorylated in living mycobacteria, recombinant $M$.

503 tuberculosis FhaA produced in M. smegmatis was digested and analyzed by nano-LC MS/MS. Three

504 FhaA phosphopeptides were found, two of them matching those previously identified in the in vitro 505 phosphorylation assay $\left(\mathrm{Thr}_{18}\right.$ in sequence 14-25 and $\mathrm{Thr}_{116}$ in sequence 108-120) (Fig. 6A and Fig. 6B, respectively; and Table S8). An additional phosphopeptide was detected corresponding to sequence 368-

507 378, with $\mathrm{Thr}_{377}$ identified as the phosphorylation site (Fig. 6C and table S8). Previous studies reported

508 that the kinase $\mathrm{PknB}$ is able to phosphorylate the FhaA residue $\mathrm{Thr}_{116}$ in vitro and several

509 phosphoproteomics reports showed that this residue is also phosphorylated in vivo [49,50]. Even when it

510 is difficult to exclude the possibility of physiologically non-relevant modifications due to protein

511 overexpression, our results suggest that PknG might also contribute to the phosphorylation of $\mathrm{Thr}_{116}$ and 512 is likely responsible for the specific phosphorylation of $\mathrm{Thr}_{18}$ in living mycobacteria.

\section{4. Discussion}


In this work we develop an AP-MS protocol for the identification of specific substrates and interactors of the mycobacterial protein kinase PknG by combining sequential elution steps and the use of different

517 PknG constructions as baits. The proposed strategy presents several advantages that allowed us to report a 518 confident list of PknG's interactors. On one hand the use of specific elution conditions (E1 and E2)

519 allowed us to fractionate PknG interactome, facilitating the detection of less abundant proteins and at the 520 same time decreasing the number of unspecific proteins eluted. On the other hand, the use PknG 521 constructions with and without the autophosphorylated sites allowed us to discern which interactions 522 relies on this docking site. Altogether, we report 66 proteins that participate in PknG-mediated protein 523 complexes in mycobacteria. One striking feature of the interactome of $\mathrm{PknG}$ is the presence of a high 524 number of ribosomal proteins and proteins related to translation. It is interesting to note that many of the 525 identified PknG interactors are predicted to be part of the RelA regulon, which mediates the stringent 526 response under nutrient limitation conditions [51] (Table S5). The presence of a large number of 527 ribosomal proteins among PknG interactors might reflect the recovery of multiprotein complexes, 528 possibly due to the simultaneous interaction of $\mathrm{PknG}$ with several specific proteins partners. Prisic et al. 529 suggested that translation was regulated by Serine/Threonine protein kinase(s), as they identified 530 phosphorylation sites in several ribosomal and ribosome-associated proteins in M. tuberculosis [5]. The 531 occurrence of phosphorylated ribosomal proteins was further confirmed by phosphoproteomic analysis in related mycobacteria $[6,52]$. However, the impact of these modifications on protein biosynthesis is still not fully understood. Ribosomal proteins are among the most common contaminants in interactomes, but they are also part of physiologically relevant macromolecular complexes [53]. However, the analysis of proteins interacting with a mock resin, using three biological replicates, indicates that these proteins are not unspecific interactors. Thus, based on the employed experimental approach, the high enrichment of

537 proteins related to translation and the available data reporting ribosomal proteins as kinases substrates 538 (including $\mathrm{PknG}$ ), we predict that ribosomal proteins are genuine partners of PknG. This conclusion is 539 also supported by a recent report that identified protein translation as one of the main processes regulated 540 by PknG [23]. Nakedi et al. used a label free phosphoproteomic approach to identify proteins that were 
541 differentially phosphorylated in wild type M. bovis BCG and a PknG knockout mutant strain, and their list 542 of 22 candidate PknG substrates include two ribosomal proteins (the 50S ribosomal protein L2 and the 54330 S ribosomal protein S16), both of which were also identified in the present work (Table S5) Overall, 544 we recovered 6 out of the 22 PknG substrates reported by Nakedi et al, namely DnaK, RNA polymerase545 binding protein $\mathrm{RbpA}$, the 50S ribosomal protein L2, the 30S ribosomal protein S16, the ATP synthase 546 beta subunit and the protein FhaA, thus strengthen the idea that these proteins represent physiological 547 substrates/interactors of the kinase. Protein microarrays have also been used to identify PknG direct 548 interactors [22,24]. Deng et al reported 59 protein interactors of $\mathrm{PknG}$, while $\mathrm{Wu}$ et al reported 122 549 interactors among which 31 were exclusive of $\mathrm{PknG}$ while the remaining 91 were also interactors of at 550 least one more protein kinase. Using the same microarray and an optimized protocol the authors increased 551 from 59 to 122 the number of interactors found, but only 21 hits were common to the lists in both papers.

552 This strategy seeks a different objective than ours; in one case direct interactors are identified while in the 553 other protein complexes that include direct and indirect interactors are analyzed. However, there is 554 strikingly little overlap between the lists of interactors found by protein microarrays and those obtained 555 by using other approaches. In particular, none of the interactors reported by Wu et al was identified in our 556 557 558 559 interactomic approach, while only one was identified by Nakedi et al (uncharacterized protein $\mathrm{A} 0 \mathrm{~A} 0 \mathrm{H} 3 \mathrm{M} 4 \mathrm{P} 0)$. Very interestingly, the strategy designed here allowed us to recover the only two previously well-characterized substrates of PknG: GarA and the 50 ribosomal protein L13, pointing to a confident list of biologically relevant interactors. None of the other reports on substrates or direct interactors of PknG succeeded in the identification of these validated substrates [22-24]. components of substrate-mediated complexes. However, we were not able to identify phosphopeptides to 564 support phosphorylation by PknG during elution. Several factors preclude phosphopeptide identification 565 in protein mixtures. On one hand, phosphopeptides have lower ionization efficiencies and ionization is 566 frequently suppressed by the presence of non-phosphorylated peptides. Additionally, phosphopeptides 
generate low quality MS/MS spectra dominated by the neutral loss of the phosphate group, resulting in lower confidence of spectral matching [54,55]. In spite of this, several pieces of evidence support the relevance of this list of putative PknG substrates. First, GarA was systematically identified in all replicates of this fraction. Additionally, 6 out of 8 proteins recovered in E1 (GarA, GS, DnaK, 60kDa chaperonin, inorganic pyrophosphatase, and Acetyl-/propionyl-coenzyme A carboxylase alpha chain) have been previously reported as kinases substrates in phosphoproteomics and/or in vitro phosphorylation assays $[7,20,30,49,56]$. Remarkably, differences in DnaK phosphorylation have been recently reported for a strain lacking PknG [23]. Finally, comparative proteomics between wild type M. tuberculosis and a $\Delta p k n G$ mutant strain using DIGE strongly suggest that two of the proteins recovered in E1, GarA and GS, are indeed differentially phosphorylated in the absence of PknG (Fig. 1).

The enzyme GS catalyzes the condensation of glutamate and ammonium to produce glutamine in the first step of nitrogen assimilation [57,58]. Overall, our results indicate that GS might represent a new substrate of PknG. First, we corroborated that GS is phosphorylated by PknG in vitro and we identified three residues as specific phosphorylation sites (Fig. 3). In addition, we provide evidence that GS is indeed phosphorylated in M. tuberculosis, and that phosphorylated proteoforms are underrepresented in a PknG null mutant (Fig. 2). Furthermore, the abundance of one of these GS proteoforms increased upon incubation of M. tuberculosis $\Delta p k n G$ protein extracts with PknG, pointing to a direct effect of PknG on

584 GS phosphorylation. Unfortunately, due to the low abundance of GS phosphorylated spots we could not confirm its phosphorylation site in vivo. Interestingly, a recent report supports the phosphorylation of GS

586 in vivo within its N-terminal sequence, including the specific phosphorylation on $\operatorname{Ser}_{57}$ [59]. Thus,

587 phosphorylation sites were assigned to $\operatorname{Ser}_{56}$ or $\operatorname{Ser}_{57}$ in the case of the sequence

$588{ }_{45}$ SVFDDGLAFDGSSIR $_{59}$ and to $\operatorname{Ser}_{63}$ or $\operatorname{Ser}_{67}$ in the case of the sequence

$589{ }_{60} \mathrm{GFQSIHESDMLLLPDPETAR}_{79}$. Moreover, these authors did not find any difference in the 590 phosphorylation status of these peptides when inhibiting PknA and PknB, indicating that other kinase(s) 591 must be responsible for these phosphorylation events. GS plays a key role in bacterial survival inside the 592 host, regulating not only ammonium assimilation but also the synthesis of the poly L-glutamate/glutamine 
cell wall structure $[58,60]$. GS synthesis and activity are tightly regulated at different levels and, in

594 particular, the reversible AMPylation of the conserved residue $\mathrm{Tyr}_{406}$ is a well-established regulatory 595 mechanism [61-63]. Our results open the possibility that protein phosphorylation also participates in the 596 modulation of GS activity; however, more experiments will be required to verify this hypothesis. Notably, 597 GS is involved in the bacterial nitrogen metabolism, similar to three other metabolic enzymes indirectly 598 regulated by PknG. It has been shown previously that the unphosphorylated form of GarA is an allosteric 599 inhibitor of alpha-ketoglutarate dehydrogenase and glutamate dehydrogenase, and an activator of 600 glutamate synthase [20,31]. PknG switches off these modulatory activities by phosphorylating GarA at $601 \mathrm{Thr}_{21}[20,31]$. Thus, GarA regulates in a very coordinated manner three of the four enzymes responsible 602 for the balance between ammonium assimilation and glutamate oxidative deamination. Strikingly, the 603 fourth enzyme that participates in this process, GS, was identified in the current study as a putative PknG 604 substrate/interactor. In this way, PknG could regulate the four key enzymes for ammonium assimilation in 605 mycobacteria through the concerted action on GarA and GS. Altogether, our results strongly suggest that 606 GS is a new substrate of PknG. However, the specific effect of GS phosphorylation on its activity, 607 oligomerization state and/or modulation by AMPylation remains to be established.

608 One of the aims of our interactomic approach was to identify PknG partners specifically interacting 609 with the kinase N-terminal docking site(s). Two proteins consistently recruited only by full-length PknG 610 contained an FHA domain: GarA and FhaA (Table 1). We demonstrated that FhaA is a substrate of PknG 611 in vitro (Fig. 5) and that the phosphorylated N-terminus of $\mathrm{PknG}$ is required for the interaction (Supp.

612 Fig. 2). The fact that FhaA was recovered in fraction E2 but not in E1 suggest that, opposite to GarA, the 613 intramolecular recognition of the pThr residues by the FHA domain in FhaA is not a favoured and 614 therefore, phosphorylation does not trigger disruption of the PknG-FhaA complex. FhaA is a two-domain 615 protein with a C-terminal FHA domain and a N-terminal domain of unknown function connected via a 616 long unstructured linker region (300 residues in M. tuberculosis) [50]. The fhaA gene is encoded by a 617 highly conserved mycobacterial operon involved in the control of cell shape and cell division [64]. A 618 previous study described the recruitment of FhaA by a phosphorylated Thr residue in the pseudokinase 
621 on Tyr, however the kinase(s) responsible(s) for each phosphorylation event are still poorly characterized.

622 [49]. Here we identified three phosphorylation sites in M. tuberculosis FhaA overproduced in $M$. smegmatis $\left(\mathrm{Thr}_{18}, \mathrm{Thr}_{116}\right.$ and $\left.\mathrm{Thr}_{377}\right)$ and two of them $\left(\mathrm{Thr}_{116}\right.$ and $\left.\mathrm{Thr}_{377}\right)$ have been previously detected in

624 vivo. In particular, previous studies reported that the kinase $\mathrm{PknB}$ is able to phosphorylate $\mathrm{Thr}_{116}$ [65]

625 while it has been proposed that PknG can phosphorylate the conserved $\mathrm{Thr}_{377}\left(\mathrm{Thr}_{371}\right.$ in M. bovis $\left.\mathrm{BCG}\right)$

626 [23]. Thus, $\mathrm{Thr}_{18}$ represents a putative new FhaA phosphorylation site. In addition, our results also

627 suggest that PknG might contribute to the phosphorylation of $\mathrm{Thr}_{116}$ in FhaA in vivo. Indeed, the

628 phosphorylation of the same substrate by different kinases, possibly in response to different stimuli, has

629 already been reported in M. tuberculosis [20,30]. We have tried to compare FhaA phosphorylation in wild

630 type $M$. tuberculosis and the $\Delta p k n G$ mutant strain by DIGE and shotgun approaches; however we failed

631 to obtain reasonable read-outs, possibly due to the low abundance of these proteins in mycobacterial

632 extracts. In spite of this, our results suggest that PknG might play a role in cell wall synthesis through

633 FhaA phosphorylation. Interestingly, Nakedi et al recently identified the FhaA orthologue in M. bovis as a

634 physiological substrate of PknG [23].

635 Altogether, we used a reliable AP-MS protocol to study the interactome of PknG and identify new 636 substrates, interactors and processes regulated by this kinase. Our results point to nitrogen and energy 637 metabolism, cell wall biosynthesis and protein translation as processes potentially modulated by PknG.

638 M. tuberculosis must reprogram its metabolism and gene expression profile in response to external

639 stimuli to survive inside the host. The accumulating evidence indicates that PknG is involved in the

640 regulation of core processes in the bacterial physiology, essential for the adaptation to the host

641 environment. Further studies on the newly reported substrates/interactors are required to test this

642 hypothesis, which warrants interesting insights about new drug targets to fight tuberculosis. 
PknG is a Serine/Threonine protein kinase recognized as a key player in mycobacterial physiology and

646 pathogenesis. The complete set of substrates and interactors that participate in signal transduction together

647 with PknG needs to be identified to understand the role of the kinase at the molecular level.

648 Using a tailored approach to study the interactome of PknG, we have identified two new substrates: the

649 enzyme glutamine synthetase and the protein FhaA. Our results show that PknG phosphorylates in vitro

650 specific residues in both glutamine synthetase and FhaA, and we provide evidence that these proteins are

651 likely phosphorylated by PknG in living mycobacteria. Our work complements previous reports on PknG

652 substrates and interactors, and reinforces a central role of PknG in the control of the bacterial nitrogen

653 metabolism. In addition, we provide evidence that PknG might regulate other processes in mycobacterial

654 physiology, including protein translation and cell wall synthesis.

655

656 Acknowledgments: This work was funded by grants from the Agencia Nacional de Investigación e

657 Innovación, Uruguay (ANII) [FCE_3_2013_1_100358 and FCE_1_2014_1_104045] and FOCEM

658 (MERCOSUR Structural Convergence Fund, COF 03/11). MG, JR and BR were supported by a

659 fellowship from ANII [POS_NAC_2012_1_8824, POS_NAC_2015_1_109755,

660 POS_FCE_2015_1_1005186] and AC was supported by Agence Nationale pour la Recherche (France)

661 [grant 09 BLAN 0400 01]. We thank M. Portela for her excellent technical support. We also thank Dr.

662 Av-Gay and Dr. S. Mowbray for kindly providing M. tuberculosis $\Delta p k n G$ strain and Rv2220 plasmid

663 respectively.

664

665 Conflict of interest: The authors declare that they have no conflicts of interest with the contents of this

666 article.

667 
668

669 [1] World Health Organization. Annual TB Report, (2017).

670

671

672

673

674

675

676

677

678

679

680

681

682

683

684

685

686

687

688

689

690

691

692

693

\section{References:}

http://www.searo.who.int/tb/documents/annual_tb_repot_2017/en/. doi:10.1038/ni.1781. (2010) 7521-6. doi:10.1073/pnas.0913482107. Microbiol. 6 (2015) 1-12. doi:10.3389/fmicb.2015.00006. (2014) 9265-9270. doi:10.1073/pnas.1323894111. Spectr. 2 (2014). doi:10.1128/microbiolspec.MGM2-0006-2013.

[2] M. Gengenbacher, S.H.E. Kaufmann, Mycobacterium tuberculosis : success through dormancy, FEMS Microbiol. Rev. 36 (2012) 514-532. doi:10.1111/j.1574-6976.2012.00331.x.

[3] D.G. Russell, P.-J. Cardona, M.-J. Kim, S. Allain, F. Altare, Foamy macrophages and the progression of the human tuberculosis granuloma, Nat. Immunol. 10 (2009) 943-948.

[4] C. Ortega, R. Liao, L.N. Anderson, T. Rustad, A.R. Ollodart, A.T. Wright, D.R. Sherman, C. Grundner, Mycobacterium tuberculosis Ser/Thr Protein Kinase B Mediates an Oxygen-Dependent Replication Switch, PLoS Biol. 12 (2014). doi:10.1371/journal.pbio.1001746.

[5] S. Prisic, S. Dankwa, D. Schwartz, M.F. Chou, J.W. Locasale, C.-M. Kang, G. Bemis, G.M. Church, H. Steen, R.N. Husson, Extensive phosphorylation with overlapping specificity by Mycobacterium tuberculosis serine/threonine protein kinases., Proc. Natl. Acad. Sci. U. S. A. 107

[6] S. Fortuin, G.G. Tomazella, N. Nagaraj, S.L. Sampson, N.C. Gey van Pittius, N.C. Soares, H.G. Wiker, G.A. de Souza, R.M. Warren, Phosphoproteomics analysis of a clinical Mycobacterium tuberculosis Beijing isolate: Expanding the mycobacterial phosphoproteome catalog, Front.

[7] U. Kusebauch, C. Ortega, A. Ollodart, R.S. Rogers, D.R. Sherman, R.L. Moritz, C. Grundner, Mycobacterium tuberculosis supports protein tyrosine phosphorylation, Proc. Natl. Acad. Sci. 111

[8] S. Prisic, R.N. Husson, Mycobacterium tuberculosis Serine/Threonine Protein Kinases, Microbiol.

[9] D.R. Sherman, C. Grundner, Agents of change - concepts in Mycobacterium tuberculosis Ser/Thr/Tyr phosphosignalling., Mol. Microbiol. 94 (2014) 231-41. doi:10.1111/mmi.12747. 
[10] S.T. Cole, R. Brosch, J. Parkhill, T. Garnier, C. Churcher, D. Harris, S. V. Gordon, K. Eiglmeier, S. Gas, C.E. Barry, F. Tekaia, K. Badcock, D. Basham, D. Brown, T. Chillingworth, R. Connor, R. Davies, K. Devlin, T. Feltwell, S. Gentles, N. Hamlin, S. Holroyd, T. Hornsby, K. Jagels, A. Krogh, J. McLean, S. Moule, L. Murphy, K. Oliver, J. Osborne, M.A. Quail, M.-A. Rajandream, J. Rogers, S. Rutter, K. Seeger, J. Skelton, R. Squares, S. Squares, J.E. Sulston, K. Taylor, S. Whitehead, B.G. Barrell, Deciphering the biology of Mycobacterium tuberculosis from the complete genome sequence, Nature. 393 (1998) 537-544. doi:10.1038/31159.

[11] Y. Av-Gay, M. Everett, The eukaryotic-like Ser/Thr protein kinases of Mycobacterium tuberculosis., Trends Microbiol. 8 (2000) 238-44. http://www.ncbi.nlm.nih.gov/pubmed/10785641.

[12] S. Cowley, M. Ko, N. Pick, R. Chow, K.J. Downing, B.G. Gordhan, J.C. Betts, V. Mizrahi, D. a Smith, R.W. Stokes, Y. Av-Gay, The Mycobacterium tuberculosis protein serine/threonine kinase PknG is linked to cellular glutamate/glutamine levels and is important for growth in vivo., Mol. Microbiol. 52 (2004) 1691-702. doi:10.1111/j.1365-2958.2004.04085.x.

[13] N.A. Kruh, J. Troudt, A. Izzo, J. Prenni, K.M. Dobos, Portrait of a pathogen: the Mycobacterium tuberculosis proteome in vivo., PLoS One. 5 (2010) e13938. doi:10.1371/journal.pone.0013938.

[14] A. Wehenkel, M. Bellinzoni, M. Graña, R. Duran, A. Villarino, P. Fernandez, G. Andre-Leroux, P. England, H. Takiff, C. Cerveñansky, S.T. Cole, P.M. Alzari, Mycobacterial Ser/Thr protein kinases and phosphatases: physiological roles and therapeutic potential., Biochim. Biophys. Acta. 1784 (2008) 193-202. doi:10.1016/j.bbapap.2007.08.006.

[15] B. Rieck, G. Degiacomi, M. Zimmermann, A. Cascioferro, F. Boldrin, N.R. Lazar-Adler, A.R. Bottrill, F. le Chevalier, W. Frigui, M. Bellinzoni, M.N. Lisa, P.M. Alzari, L. Nguyen, R. Brosch, U. Sauer, R. Manganelli, H.M. O’Hare, PknG senses amino acid availability to control metabolism and virulence of Mycobacterium tuberculosis, 2017. doi:10.1371/journal.ppat.1006399.

[16] J. Walburger, A., Koul, A., Nguyen, L., Prescianotto-Baschong, C., Huygen, K., Klebl, B., Thompson, C., Bacher, C., Pieters, Protein Kinase G from Pathogenic Mycobacteria Promotes 
Survival Within Macrophages, Science (80-. ). 304 (2004) 1800-1804. doi:10.1126/science.1099384.

[17] M.Z. Khan, A. Bhaskar, S. Upadhyay, P. Kumari, R.S. Rajmani, P. Jain, A. Singh, D. Kumar, N.S. Bhavesh, V.K. Nandicoori, Protein kinase G confers survival advantage to Mycobacterium tuberculosis during latency-like conditions., J. Biol. Chem. 292 (2017) 16093-16108. doi:10.1074/jbc.M117.797563.

[18] R. Paroha, R. Chourasia, R. Mondal, S.K. Chaurasiya, PknG supports mycobacterial adaptation in acidic environment, Mol. Cell. Biochem. (2017) 1-12. doi:10.1007/s11010-017-3211-x.

[19] K. a Wolff, H.T. Nguyen, R.H. Cartabuke, A. Singh, S. Ogwang, L. Nguyen, Protein kinase G is required for intrinsic antibiotic resistance in mycobacteria., Antimicrob. Agents Chemother. 53 (2009) 3515-9. doi:10.1128/AAC.00012-09.

[20] H.M. O’Hare, R. Durán, C. Cerveñansky, M. Bellinzoni, A.M. Wehenkel, O. Pritsch, G. Obal, J. Baumgartner, J. Vialaret, K. Johnsson, P.M. Alzari, Regulation of glutamate metabolism by protein kinases in mycobacteria., Mol. Microbiol. 70 (2008) 1408-23. doi:10.1111/j.13652958.2008.06489.x.

[21] K.A. Wolff, A.H. de la Peña, H.T. Nguyen, T.H. Pham, L.M. Amzel, S.B. Gabelli, L. Nguyen, A Redox Regulatory System Critical for Mycobacterial Survival in Macrophages and Biofilm Development, PLoS Pathog. 11 (2015) 1-20. doi:10.1371/journal.ppat.1004839.

[22] J. Deng, L. Bi, L. Zhou, S. Guo, J. Fleming, H. Jiang, Y. Zhou, J. Gu, Q. Zhong, Z. Wang, Z. Liu, R. Deng, J. Gao, T. Chen, W. Li, J. Wang, X. Wang, H. Li, F. Ge, G. Zhu, H. Zhang, J. Gu, F. Wu, Z. Zhang, D. Wang, H. Hang, Y. Li, L. Cheng, X. He, S. Tao, X. Zhang, Mycobacterium tuberculosis proteome microarray for global studies of protein function and immunogenicity., Cell Rep. 9 (2014) 2317-29. doi:10.1016/j.celrep.2014.11.023.

[23] K.C. Nakedi, B. Calder, M. Barnejee, A. Giddey, A.J. Nel, S. Garnett, J.M. Blackburn, N.A. Da Cruz Soares, Identification of novel physiological substrates of Mycobacterium Bovis BCG Protein Kinase G (PknG) by label-free quantitative phosphoproteomics., Mol. Cell. Proteomics. 
(2018) mcp.RA118.000705. doi:10.1074/mcp.RA118.000705.

[24] F.-L. Wu, Y. Liu, H.-W. Jiang, Y. Luan, H. Zhang, X. He, Z.-W. Xu, J.-L. Hou, L.-Y. Ji, Z. Xie, D.M. Czajkowsky, W. Yan, J.-Y. Deng, L.-J. Bi, X.-E. Zhang, S.-C. Tao, The Ser / Thr Protein Kinase Protein-Protein Interaction Map of M . tuberculosis, Mol. Cell. Proteomics. 16 (2017) 142. doi:10.1074/mcp.M116.065771.

[25] M. Gil, M. Graña, F.J. Schopfer, T. Wagner, A. Denicola, B.A. Freeman, P.M. Alzari, C. Batthyány, R. Durán, R.D. M. Gil, M. Graña, F. J. Schopfer, T. Wagner, A. Denicola, B. A. Freeman, P. M. Alzari, C. Batthyány, Inhibition of Mycobacterium tuberculosis PknG by noncatalytic rubredoxin domain specific modification: reaction of an electrophilic nitro-fatty acid with the Fe-S center, Free Radic. Biol. Med. 65 (2013) 150-161. doi:10.1038/jid.2014.371.

[26] M.N. Lisa, M. Gil, G. André-Leroux, N. Barilone, R. Durán, R.M. Biondi, P.M. Alzari, Molecular Basis of the Activity and the Regulation of the Eukaryotic-like S/T Protein Kinase PknG from Mycobacterium tuberculosis, Structure. 23 (2015) 1039-1048. doi:10.1016/j.str.2015.04.001.

[27] N. Scherr, S. Honnappa, G. Kunz, P. Mueller, R. Jayachandran, F. Winkler, J. Pieters, M.O. Steinmetz, Structural basis for the specific inhibition of protein kinase G, a virulence factor of Mycobacterium tuberculosis., Proc. Natl. Acad. Sci. U. S. A. 104 (2007) 12151-6. doi:10.1073/pnas.0702842104.

[28] D. Durocher, S.P. Jackson, The FHA domain, FEBS Lett. 513 (2002) 58-66. doi:10.1016/S00145793(01)03294-X.

[29] P. England, A. Wehenkel, S. Martins, S. Hoos, G. André-Leroux, A. Villarino, P.M. Alzari, The FHA-containing protein GarA acts as a phosphorylation-dependent molecular switch in mycobacterial signaling, FEBS Lett. 583 (2009) 301-307. doi:10.1016/j.febslet.2008.12.036.

[30] A. Villarino, R. Duran, A. Wehenkel, P. Fernandez, P. England, P. Brodin, S.T. Cole, U. ZimnyArndt, P.R. Jungblut, C. Cerveñansky, P.M. Alzari, Proteomic identification of M. tuberculosis protein kinase substrates: PknB recruits GarA, a FHA domain-containing protein, through activation loop-mediated interactions., J. Mol. Biol. 350 (2005) 953-63. 
doi:10.1016/j.jmb.2005.05.049.

[31] T.J. Nott, G. Kelly, L. Stach, J. Li, S. Westcott, D. Patel, D.M. Hunt, S. Howell, R.S. Buxton, H.M. O’Hare, S.J. Smerdon, An Intramolecular Switch Regulates Phosphoindependent FHA Domain Interactions in Mycobacterium tuberculosis, Sci. Signal. 2 (2009) ra12-ra12. doi:10.1126/scisignal.2000212.

[32] J.C. van Kessel, L.J. Marinelli, G.F. Hatfull, Recombineering mycobacteria and their phages, Nat. Rev. Microbiol. 6 (2008) 851-857. doi:10.1038/nrmicro2014.

[33] W.W. Krajewski, T.A. Jones, S.L. Mowbray, Structure of Mycobacterium tuberculosis glutamine synthetase in complex with a transition-state mimic provides functional insights, Proc. Natl. Acad. Sci. 102 (2005) 10499-10504. doi:10.1073/pnas.0502248102.

[34] A. Shevchenko, M. Wilm, O. Vorm, M. Mann, Mass Spectrometric Sequencing of Proteins from Silver-Stained Polyacrylamide Gels Mass Spectrometric Sequencing of Proteins from SilverStained Polyacrylamide Gels, Anal. Chem. 68 (1996) 850-858. doi:10.1021/ac950914h.

[35] P.C. Carvalho, J.S.G. Fischer, T. Xu, J.R. Yates, V.C. Barbosa, PatternLab: From mass spectra to label-free differential shotgun proteomics, Curr. Protoc. Bioinforma. (2012) 1-18. doi:10.1002/0471250953.bi1319s40.

[36] P.C. Carvalho, D.B. Lima, F. V Leprevost, M.D.M. Santos, J.S.G. Fischer, P.F. Aquino, J.J. Moresco, J.R. Yates, V.C. Barbosa, J.R.Y. Iii, V.C. Barbosa, Integrated analysis of shotgun proteomic data with PatternLab for proteomics 4.0, Nat. Protoc. 11 (2016) 102-117. doi:10.1038/nprot.2015.133.

[37] P.C. Carvalho, J.S.G. Fischer, J. Perales, J.R. Yates, V.C. Barbosa, E. Bareinboim, Analyzing marginal cases in differential shotgun proteomics., Bioinformatics. 27 (2011) 275-6. doi:10.1093/bioinformatics/btq632.

[38] A. Kapopoulou, J.M. Lew, S.T. Cole, The MycoBrowser portal: A comprehensive and manually annotated resource for mycobacterial genomes, Tuberculosis. 91 (2011) 8-13. doi:10.1016/j.tube.2010.09.006. 
[39] W. Gish, D.J. States, Identification of protein coding regions by database similarity search, Nat Genet. 3 (1993) 266-272. doi:10.1038/ng0393-266.

[40] D. Szklarczyk, A. Franceschini, S. Wyder, K. Forslund, D. Heller, J. Huerta-Cepas, M. Simonovic, A. Roth, A. Santos, K.P. Tsafou, M. Kuhn, P. Bork, L.J. Jensen, C. Von Mering, STRING v10: Protein-protein interaction networks, integrated over the tree of life, Nucleic Acids Res. 43 (2015) D447-D452. doi:10.1093/nar/gku1003.

[41] H. Mi, S. Poudel, A. Muruganujan, J.T. Casagrande, P.D. Thomas, PANTHER version 10: Expanded protein families and functions, and analysis tools, Nucleic Acids Res. 44 (2016) D336D342. doi:10.1093/nar/gkv1194.

[42] J.A. Vizcaino, A. Csordas, N. Del-Toro, J.A. Dianes, J. Griss, I. Lavidas, G. Mayer, Y. PerezRiverol, F. Reisinger, T. Ternent, Q.W. Xu, R. Wang, H. Hermjakob, 2016 update of the PRIDE database and its related tools, Nucleic Acids Res. 44 (2016) D447-D456. doi:10.1093/nar/gkv1145.

[43] R. Mehta, J.T. Pearson, S. Mahajan, A. Nath, M.J. Hickey, D.R. Sherman, W.M. Atkins, Adenylylation and catalytic properties of Mycobacterium tuberculosis glutamine synthetase expressed in Escherichia coli versus mycobacteria., J. Biol. Chem. 279 (2004) 22477-82. doi:10.1074/jbc.M401652200.

[44] B.M. Shapiro, H.S. Kingdon, E.R. Stadtman, Regulation of glutamine synthetase. VII. Adenylyl glutamine synthetase: a new form of the enzyme with altered regulatory and kinetic properties., Proc. Natl. Acad. Sci. U. S. A. 58 (1967) 642-9. http://www.ncbi.nlm.nih.gov/pubmed/4860756 (accessed April 25, 2018).

[45] C. Grundner, L.M. Gay, T. Alber, Mycobacterium tuberculosis serine/threonine kinases PknB, PknD, PknE, and PknF phosphorylate multiple FHA domains., Protein Sci. 14 (2005) 1918-1921. doi:10.1110/ps.051413405.

[46] M. Gupta, A. Sajid, G. Arora, V. Tandon, Y. Singh, Forkhead-associated domain-containing protein Rv0019c and polyketide-associated protein PapA5, from substrates of serine/threonine 
protein kinase PknB to interacting proteins of mycobacterium tuberculosis, J. Biol. Chem. 284 (2009) 34723-34734. doi:10.1074/jbc.M109.058834.

[47] V. Molle, D. Soulat, J.M. Jault, C. Grangeasse, A.J. Cozzone, J.F. Prost, Two FHA domains on an ABC transporter, Rv1747, mediate its phosphorylation by PknF, a Ser/Thr protein kinase from Mycobacterium tuberculosis, FEMS Microbiol. Lett. 234 (2004) 215-223. doi:10.1016/j.femsle.2004.03.033.

[48] K. Sharma, M. Gupta, A. Krupa, N. Srinivasan, Y. Singh, EmbR, a regulatory protein with ATPase activity, is a substrate of multiple serine/threonine kinases and phosphatase in Mycobacterium tuberculosis., FEBS J. 273 (2006) 2711-21. doi:10.1111/j.17424658.2006.05289.x.

[49] B. Calder, C. Albeldas, J.M. Blackburn, N.C. Soares, Mass spectrometry offers insight into the role of ser/thr/tyr phosphorylation in the mycobacteria, Front. Microbiol. 7 (2016) 1-8. doi:10.3389/fmicb.2016.00141.

[50] C. Roumestand, J. Leiba, N. Galophe, E. Margeat, A. Padilla, Y. Bessin, P. Barthe, V. Molle, M. Cohen-Gonsaud, Structural insight into the Mycobacterium tuberculosis Rv0020c protein and its interaction with the PknB kinase., Structure. 19 (2011) 1525-34. doi:10.1016/j.str.2011.07.011.

[51] J.L. Dahl, C.N. Kraus, H.I.M. Boshoff, B. Doan, K. Foley, D. Avarbock, G. Kaplan, V. Mizrahi, H. Rubin, C.E. Barry, The role of RelMtb-mediated adaptation to stationary phase in long-term persistence of Mycobacterium tuberculosis in mice., Proc. Natl. Acad. Sci. U. S. A. 100 (2003) 10026-31. doi:10.1073/pnas.1631248100.

[52] R. Verma, S.M. Pinto, A.H. Patil, J. Advani, P. Subba, M. Kumar, J. Sharma, G. Dey, R. Ravikumar, S. Buggi, P. Satishchandra, K. Sharma, M. Suar, S.P. Tripathy, D.S. Chauhan, H. Gowda, A. Pandey, S. Gandotra, T.S.K. Prasad, Quantitative Proteomic and Phosphoproteomic Analysis of H37Ra and H37Rv Strains of Mycobacterium tuberculosis, J. Proteome Res. 16 (2017) 1632-1645. doi:10.1021/acs.jproteome.6b00983.

[53] J.S. Rees, N. Lowe, I.M. Armean, J. Roote, G. Johnson, E. Drummond, H. Spriggs, E. Ryder, S. 
Russell, D.S. Johnston, K.S. Lilley, In Vivo Analysis of Proteomes and Interactomes Using Parallel Affinity Capture (iPAC) Coupled to Mass Spectrometry, Mol. Cell. Proteomics. 10 (2011) M110.002386-M110.002386. doi:10.1074/mcp.M110.002386.

[54] N. Dephoure, K.L. Gould, S.P. Gygi, D.R. Kellogg, Mapping and analysis of phosphorylation sites: a quick guide for cell biologists, Mol. Biol. Cell. 24 (2013) 535-542. doi:10.1091/mbc.E1209-0677.

[55] M. Mann, S. Ong, M. Gr, H. Steen, O.N. Jensen, A. Pandey, Analysis of protein phosphorylation using mass spectrometry: deciphering the phosphoproteome., Trends Biotechnol. 20 (2002) 261-8. doi:10.1016/S0167-7799(02)01944-3.

[56] M.J. Canova, R. Veyron-Churlet, I. Zanella-Cleon, M. Cohen-Gonsaud, A.J. Cozzone, M. Becchi, L. Kremer, V. Molle, The Mycobacterium tuberculosis serine/threonine kinase PknL phosphorylates Rv2175c: Mass spectrometric profiling of the activation loop phosphorylation sites and their role in the recruitment of Rv2175c, Proteomics. 8 (2008) 521-533. doi:10.1002/pmic.200700442.

[57] A. Gouzy, Y. Poquet, O. Neyrolles, Nitrogen metabolism in Mycobacterium tuberculosis physiology and virulence, Nat. Rev. Microbiol. 12 (2014) 729-737. doi:10.1038/nrmicro3349.

[58] M. V Tullius, G. Harth, M. a Horwitz, Glutamine Synthetase GlnA1 Is Essential for Growth of Mycobacterium tuberculosis in Human THP-1 Macrophages and Guinea Pigs Glutamine Synthetase GlnA1 Is Essential for Growth of Mycobacterium tuberculosis in Human THP-1 Macrophages and Guinea Pigs, Infect. Immun. 71 (2003) 3927-3936. doi:10.1128/IAI.71.7.3927.

[59] X. Carette, J. Platig, D.C. Young, M. Helmel, A.T. Young, Z. Wang, L.-P. Potluri, C.S. Moody, J. Zeng, S. Prisic, J.N. Paulson, J. Muntel, A.V.R. Madduri, J. Velarde, J.A. Mayfield, C. Locher, T. Wang, J. Quackenbush, K.Y. Rhee, D.B. Moody, H. Steen, R.N. Husson, Multisystem Analysis of Mycobacterium tuberculosis Reveals Kinase-Dependent Remodeling of the PathogenEnvironment Interface., MBio. 9 (2018) e02333-17. doi:10.1128/mBio.02333-17.

[60] G. Harth, M. a Horwitz, An inhibitor of exported Mycobacterium tuberculosis glutamine 
synthetase selectively blocks the growth of pathogenic mycobacteria in axenic culture and in human monocytes: extracellular proteins as potential novel drug targets., J. Exp. Med. 189 (1999) 1425-1436. doi:10.1084/jem.189.9.1425.

[61] P. Carroll, C.A. Pashley, T. Parish, Functional analysis of GlnE, an essential adenylyl transferase in Mycobacterium tuberculosis, J. Bacteriol. 190 (2008) 4894-4902. doi:10.1128/JB.00166-08.

[62] J.A. Leigh, J.A. Dodsworth, Nitrogen Regulation in Bacteria and Archaea, Annu. Rev. Microbiol. 61 (2007) 349-377. doi:10.1146/annurev.micro.61.080706.093409.

[63] E.R. Stadtman, The Story of Glutamine Synthetase Regulation, J. Biol. Chem. 276 (2001) 44357-

[64] C.C.L. Gee, K.G.K. Papavinasasundaram, S.R. Blair, C.E. Baer, A.M. Falick, D.S. King, J.E. Griffin, H. Venghatakrishnan, A. Zukauskas, J.-R. Wei, R.K. Dhiman, D.C. Crick, E.J. Rubin,

C.M. Sassetti, T. Alber, A phosphorylated pseudokinase complex controls cell wall synthesis in mycobacteria., Sci. Signal. 5 (2012) 1-24. doi:10.1126/scisignal.2002525. Cohen-Gonsaud, Structural insight into the Mycobacterium tuberculosis Rv0020c protein and its interaction with the PknB kinase, Structure. 19 (2011) 1525-1534. doi:10.1016/j.str.2011.07.011. 
894 Table 1. Proteins identified in E1 and E2 fractions from the AP-MS experiment.

Proteins identified as exclusive interactors of full length PknG in E1

\begin{tabular}{|c|c|c|}
\hline UniProt identifier & Description & Gene name \\
\hline A0QYG2 & Glycogen accumulation regulator GarA & MSMEG_3647 \\
\hline A0R079 & Glutamine synthetase & MSMEG_4290 \\
\hline \multicolumn{3}{|c|}{ Proteins identified as common interactors of PknG and $\mathrm{PknG}_{\Delta 73}$ in E1 } \\
\hline UniProt identifier & Description & Gene name \\
\hline A0QQC8 & Chaperone protein DnaK & MSMEG_0709 \\
\hline A0R5M3 & Alcohol dehydrogenase, iron containing & MSMEG_6242 \\
\hline A0QQU5 & $60 \mathrm{kDa}$ chaperonin 1 & MSMEG_0880 \\
\hline A0R2I1 & $4 \mathrm{Fe}-4 \mathrm{~S}$ ferredoxin, iron-sulfur binding protein & MSMEG_5122 \\
\hline A0R597 & Inorganic pyrophosphatase & MSMEG_6114 \\
\hline A0QTE1 & Acetyl-/propionyl-coenzyme A carboxylase alpha chain & MSMEG_1807 \\
\hline
\end{tabular}

Proteins identified as exclusive interactors of full length PknG in E2

\begin{tabular}{lcc}
\hline UniProt identifier & Description & Gene name \\
A0QNG7 & FHA domain protein & MSMEG_0035 \\
\hline
\end{tabular}

895

896 
Figure 1. Analysis of the differential proteoform-patterns present in wild type M. tuberculosis and a PknG null mutant by DIGE.

901 A. Representative DIGE in pseudocolours. Protein spots over-represented in WT and $\Delta p k n G$ strains are 902 shown in green and red, respectively. Differential spots with patterns consistent with changes in protein 903 phosphorylation are labelled. Protein spots in these areas were assigned to glutamine synthetase (GS,) and 904 GarA. Average standardized abundance fold changes and protein identification details for each spot are 905 shown in Table S7. B. Bar graphs showing the average standardized abundance fold changes of GarA 906 spots (spots 1-5, upper panel) and GS spots (spots 6-13, lower panel). The estimated pI range of each group of spots is indicated below each graph. Fold changes for spots 1-12 have $\mathrm{p}<0.05$ values.

Figure 2. Confirmation of GS phosphorylation status. A. DIA analysis of wild type M. tuberculosis protein extracts with or without treatment with protein phosphatase. B. DIA analysis of M. tuberculosis $\Delta p k n G$ protein extracts with or without pre-incubation with PknG under phosphorylation conditions.

912 Spots labels refers to spots in DIGE gel in Fig. 4, all identified as GS by MS; spots 6-8 were not 913 completely resolved in this DIA gel, therefore, they were analyzed as a single spot. Spots $6-8 \log _{10}$

914 volume ratio $(\mathrm{WT} / \mathrm{WT}+$ phosphatase $)=1.65$, Mascot protein score: 267 ; spot $9 \log _{10}$ volume ratio

$915(\mathrm{WT} / \mathrm{WT}+$ phosphatase $)=1.53$, Mascot protein score: 149 ; spot 9 volume ratio $(\Delta p k n G / \Delta p k n G+\mathrm{PknG})=$ 916 1.32, Mascot protein score: 111 . Volume ratios are expressed as $10 . \log _{10}$ (spot volume condition $1 /$ spot 917 volume condition 2). C-D. 3D view of the GS spot 9 showing the spot volume differences between each 918 pair of conditions.

Figure 3. Identification of GS phosphorylation sites. In each case, C-terminal (blue) and N-terminal (red) fragment ions assigned to the sequence are indicated. Ions presenting the neutral loss characteristic of phosphorylation are indicated. A. Representative MS/MS spectrum of the doubly charged peptide 
924 phosphorylated residue within the sequence (pRS score of 114; pRS site probablitiy $\mathrm{S}_{57}: 99.8 \%$; Ser 56 :

$9250.2 \%$ ). The presence of $\mathrm{y}_{3}{ }^{+}$fragment ion points to $\mathrm{S}_{57}$ and not $\mathrm{S}_{56}$ as the phosphoresidue. B.

926 Representative MS/MS spectrum of doubly charged ion of sequence GFQSIHESDMLLLPDPEpT ${ }_{77} \mathrm{AR}$

927 ( $\mathrm{m} / z$ observed 1168.2; Xcorr: 4.34). PhoshpoRS indicates that $\operatorname{Thr}_{77}$ is the phosphorylated residue within

928 the sequence (pRS score of 100; pRS probablitiy $\mathrm{T}_{77} 100 \%$ ). The presence of many y ion fragments from

$929 \mathrm{y}^{+}$to $\mathrm{y}^{+}$, either containing the +80 modification and/or the phosphate neutral loss corroborates the

930 assignment of $\operatorname{Thr}_{77}$ and not $\mathrm{S}_{67}$ or $\mathrm{S}_{63}$ as the posphoresidue. C. Representative MS/MS spectrum of triply

931 charged ion of sequence DLYELPPEEAASIPQTPpT ${ }_{421}$ QLSDVIDR ( $\mathrm{m} / \mathrm{z}$ observed: 993.1 ; Xcorr: 4.06)

932 and pRS score 58. PhosphoRS indicates that the most probably modified residue is $\mathrm{Thr}_{421}$ (pRS

933 probability 90.5\%). However the site is difficult to identify by manual inspection. D. MS/MS spectrum of

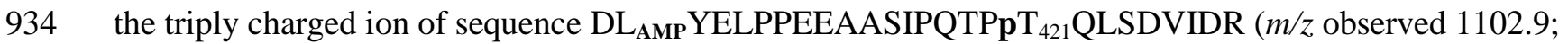

935 Xcorr 5.05) showing that both the AMPylation of $\operatorname{Tyr}_{406}$ [43] residue and the phosphorylation of a

936 residue within this sequence, most probably $\mathrm{Thr}_{421}$, can take place in the same peptide (pRS site

937 probability for Thr421 92.8\%). The presence of the fragment ions $\mathrm{y}_{10}{ }^{+}, \mathrm{y}_{10}{ }^{+}-\mathrm{H}_{3} \mathrm{PO}_{4}$; and a small signal

938 corresponding to $\mathrm{y}_{9}{ }^{+}-\mathrm{H}_{3} \mathrm{PO}_{4}$ point to $\mathrm{Thr}_{421}$ and not $\mathrm{Thr}_{420}$ as the phosphorylated residue. Additional

939 information regarding each phosphosite identification is depicted in Supplementary Table S8.

Figure 4. Location of phosphorylatable residues on GS structure. Crystal structure of the GS dodecameric form (PDB code 2BVC [33]) highlighting the three phosphorylatable sites ( $\operatorname{Ser}_{57}, \mathrm{Thr}_{77}$ and and molecules in the substrate-binding pocket are shown in sticks. Right: view of the interfacial plane

946 between the two GS 6-mer rings perpendicular to the protein 6-fold axis; center: lateral view of the GS 947 dodecamer; left: amplification of the lateral view of the GS dodecamer. 

experiments using PknG as kinase and digested previous to MS analysis. y- (blue) and b- (red) ions assigned to the sequence are indicated. Ions presenting the neutral loss characteristic of phosphorylation are shown. A. Representative MS/MS spectrum of doubly charged ion of sequence FEQSSNLHpT $\mathrm{T}_{116} \mathrm{GQFR}$ (observed $\mathrm{m} / \mathrm{z}$ 816.2, Xcorr 3.90). pRS score of 110 and pRS probability $100 \%$ for $\mathrm{T}_{116}$ allowed the unequivocal identification of the phosphorylation site. The presence of several y ions in the spectrum confirms this assignment $\left(\mathrm{y}_{5}^{+} ; \mathrm{y}_{6}^{+} ; \mathrm{y}_{6}^{+}-\mathrm{H}_{3} \mathrm{PO}_{4} ; \mathrm{y}_{7}^{+} ; \mathrm{y}_{8}^{+}\right)$

B. Representative MS/MS spectrum of triply charged ion of sequence KLEQpT ${ }_{18} \operatorname{VGDAFAR}(m / z, 472.3$, Xcorr value of 4.31). pRS score of 79 and pRS probability $100 \%$ for $\mathrm{T}_{18}$ allowed to unequivocally identify the phosphoresidue, which is confirmed by the presence of $b$ ions from $b_{5}$ to $b_{11}$.

Figure 6. Identification of FhaA phosphorylation sites in vivo. Strep-tag ${ }^{\circledR}$ II-FhaA was purified from M. smegmatis and digested previous to mass spectrometry analysis. $\mathrm{y}$ - (blue) and b- (red) fragment ions assigned to the sequence are indicated. Ions presenting the neutral loss characteristic of phosphorylation are also shown. A. Representative MS/MS spectrum of doubly charged ion of sequence FEQSSNLHpT $_{116}$ GQFR ( $\mathrm{m} / \mathrm{z}$ observed: 816.1 ; Xcorr: 4.35). Analysis using PhosphoRS indicates that $\mathrm{T}_{116}$ is the phosphorylated residue within the sequence (pRS score of 106; $\mathrm{pRS}$ probablitiy $\mathrm{T}_{116} 100 \%$ ). Manual inspection of the spectra revealed the presence of fragment ions that allow confirming the phosphorylation of $\mathrm{T}_{116}$ and not $\mathrm{S}_{111}$ or $\left.\mathrm{S}_{112}: \mathrm{y}_{5}{ }^{+} ; \mathrm{y}_{6} ;{ }^{+} \mathrm{y}_{6}{ }^{+}-\mathrm{H}_{3} \mathrm{PO}_{4 ;} \mathrm{y}_{7}{ }^{+} ; \mathrm{y}_{8}{ }^{+}\right)$. B. Representative MS/MS spectrum of triply charged ion of sequence KLEQpT ${ }_{18}$ VGDAFAR (observed $m / z 472.1$; Xcorr value of 4.25). Both phosphoRS and manual inspection of the spectra fully supports the phosphorylation of $\mathrm{T}_{18}$ within the sequence (pRS score of 84; pRS probability 100\%). C. Representative MS/MS spectrum of the 972 doubly charged ion of sequence QDYGGGADYpT ${ }_{377} \mathrm{R}$ (observed $\mathrm{m} / \mathrm{z}$ 641.9; Xcorr value of 3.54).

973 PhosphoRS analysis point to $\mathrm{T}_{377}$ as the in vivo phosphorylation site (pRS score of 91; pRS probability 
$97499.5 \%$ for $\mathrm{T}_{377}$ ). The presence of $\mathrm{y}_{2}{ }^{+}$and $\mathrm{y}_{2}{ }^{+}-\mathrm{H}_{3} \mathrm{PO}_{4}$ fragment ions confirms the phosphorylation of the $975 \operatorname{Thr}_{377}$, and not the $\mathrm{Tyr}_{376}$ residue.

976

977 Supporting information:

978

Supplementary Figures Legends

980

Supplementary Figure 1. Kinase activity of immobilized PknG. A. PknG autophosphorylation. Linear

982 MALDI-TOF spectra of tryptic digestion of immobilized PknG (upper panel) and immobilized PknG

983 previously incubated with ATP under phosphorylation conditions (bottom panel). Non-phosphorylated

984 sequence 10-60 (theoretical $\mathrm{m} / \mathrm{z}$ 5395.7), and its mono and diphosphorylated forms are indicated

985 (theoretical $\mathrm{m} / \mathrm{z} 5475.7$ and 5555.7, respectively). While the predominant signal in recombinant PknG

986 spectrum corresponds to the unphosphorylated peptide, the diphosphorylated specie is the most intense

987 ion of sequence 10-60 after autophosphorylation. B. GarA phosphorylation by PknG. Linear MALDI-

988 TOF mass spectra of recombinant GarA $(\mathrm{m} / \mathrm{z}$ 20446, black line) and GarA phosphorylated by PknG

989 immobilized on NHS-Activated Sepharose 4 Fast Flow $(m / z, 20526$, grey line). The mass shift

990 corresponds to the incorporation of one phosphate group (80 Da).

991

992 Supplementary Figure 2. PknG interacts with FhaA. FhaA was immobilized on a CM5 sensorchip by 993 standard amine coupling. Phosphorylated $\mathrm{PknG}$ and $\mathrm{PknG}_{\Delta 73}$ were diluted to a final concentration of 30 $994 \mathrm{nM}$ and injected during $180 \mathrm{~s}$ over the immobilized and a reference surfaces.

995

996 Supplementary Tables

997 Supplementary Table S1: Proteins identified in E1.

998 Supplementary Table S2: Proteins identified in E1: reported phosphosites in mycobacterial

999 phosphoproteomic analysis. 
1000 Supplementary Table S3: Proteins identified in E2.

1001 Supplementary Table S4: Proteins identified in E3.

1002 Supplementary Table S5: List of proteins identified in PknG interactome.

1003 Supplementary Table S6: Analysis of protein-protein interaction enrichment (STRING, http://string-

1004 db.org/cgi/network.pl). Overrepresentation biological processes enrichment test (PANTHER,

1005 http://www.pantherdb.org/).

1006 Supplementary Table S7: Identification of protein spots from DIGE analysis.

1007 Supplementary Table S8. Phosphorylation sites reported in this work.

1008

1009 

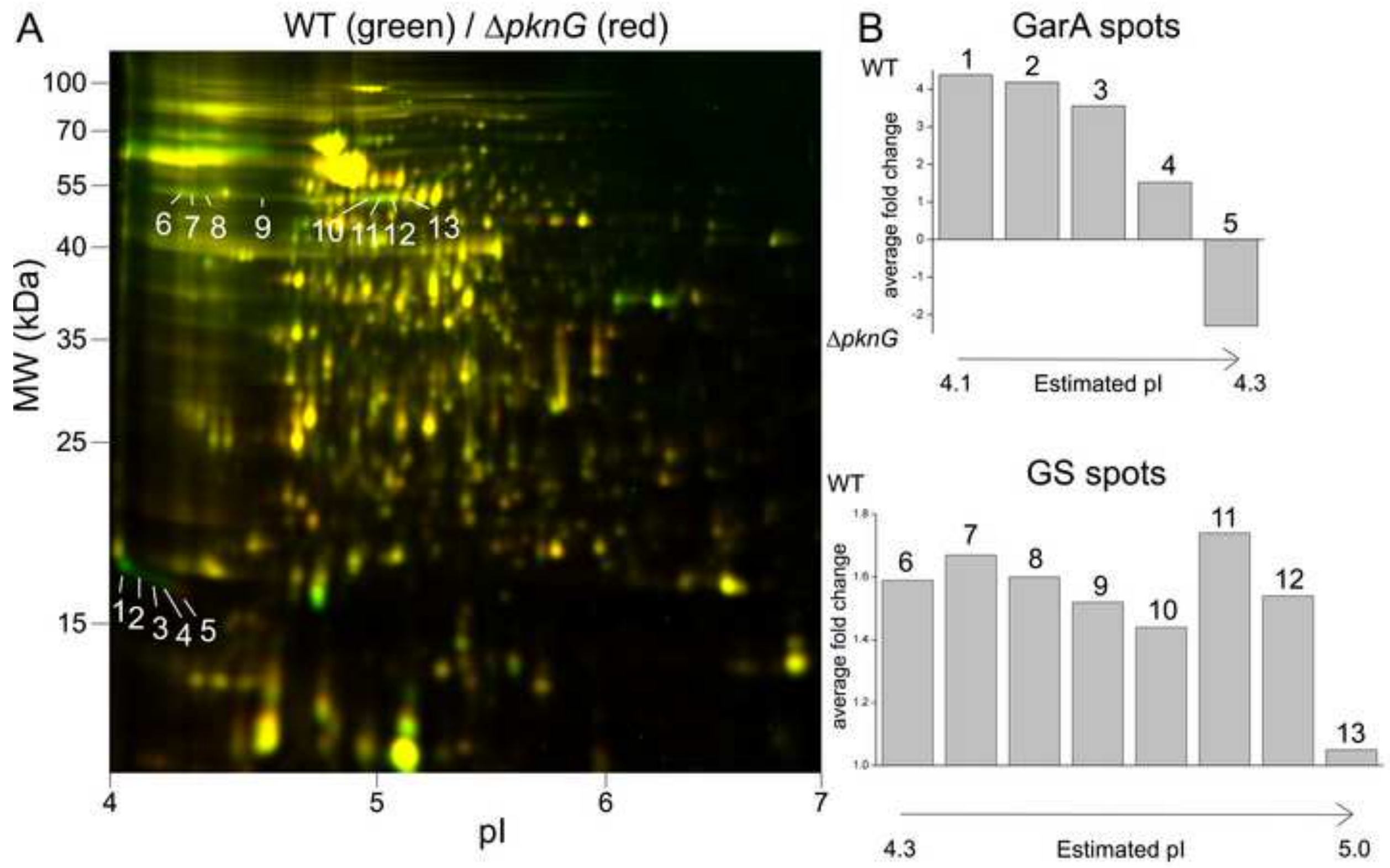


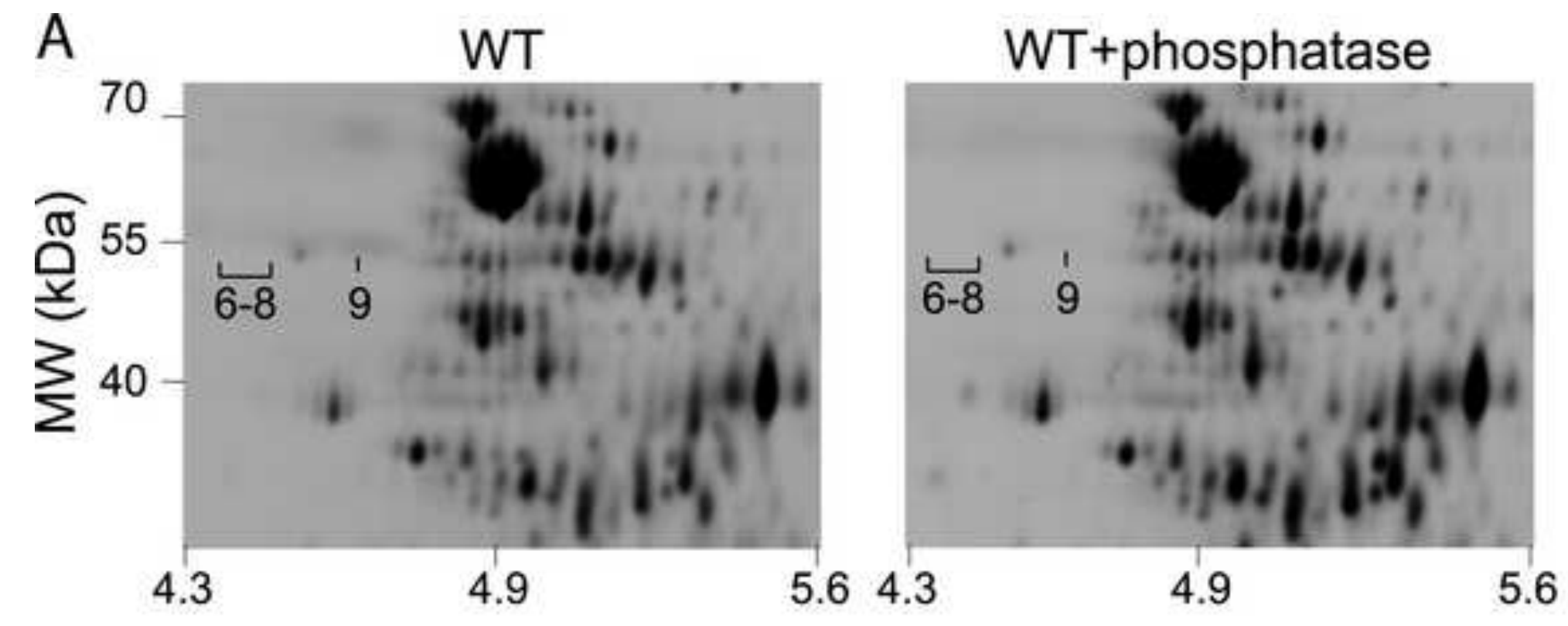

C WT

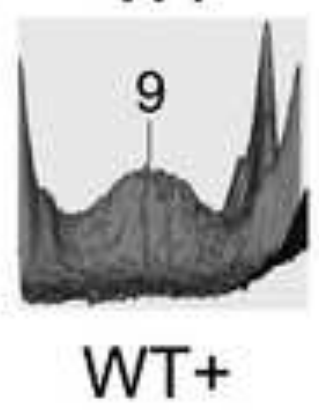

phosphatase

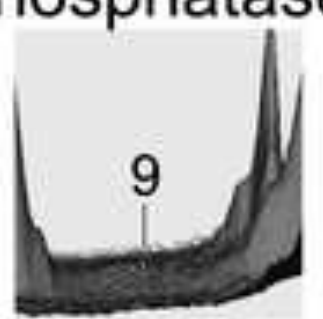

B

$\Delta p k n G$

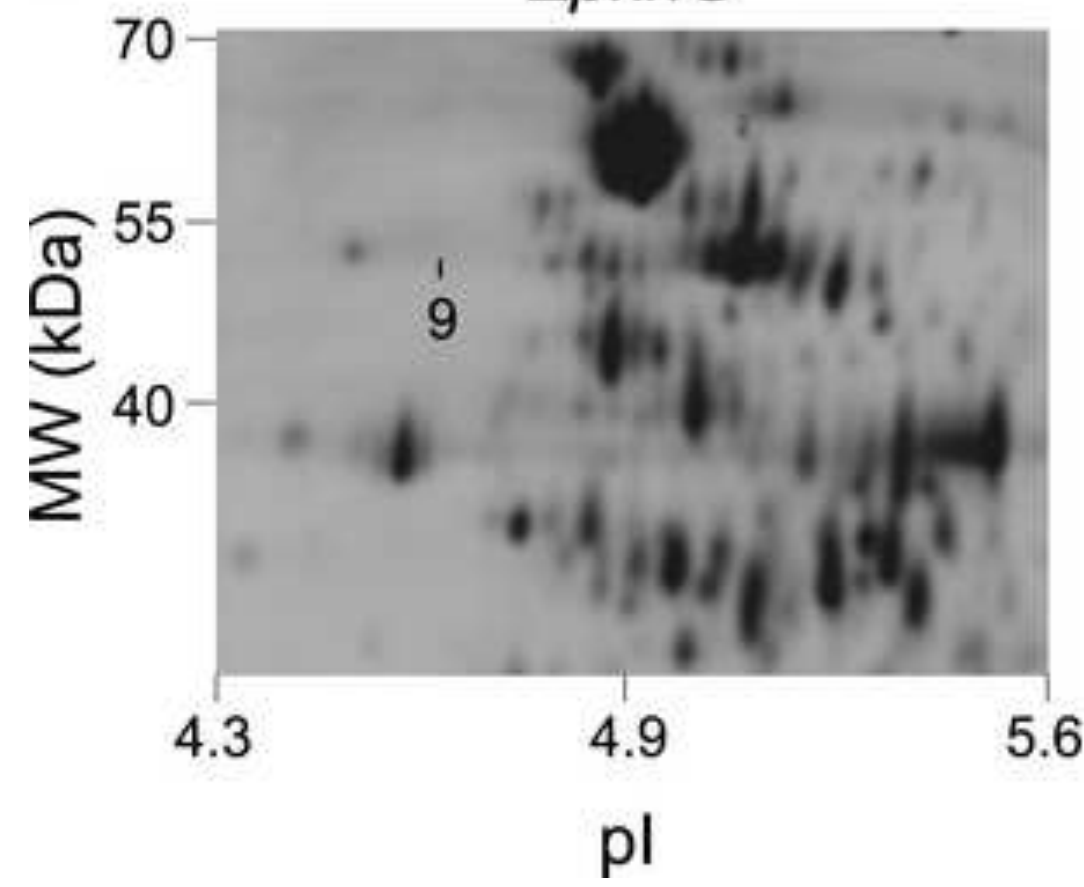

$\Delta p k n G+P k n G$

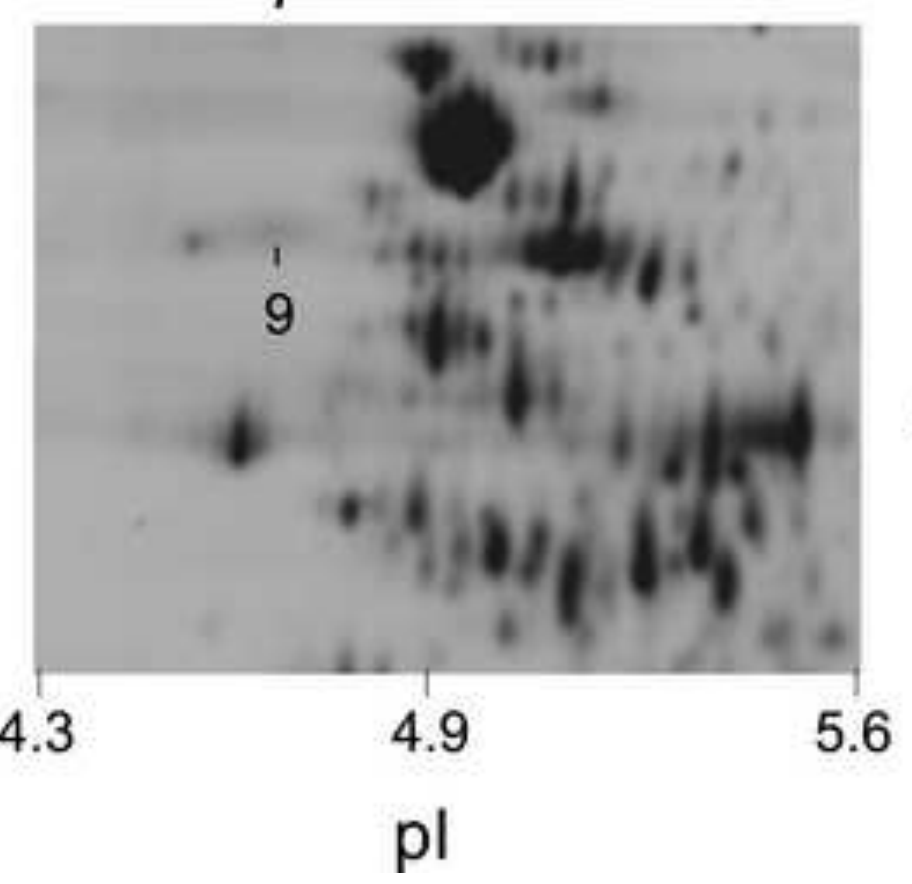

D

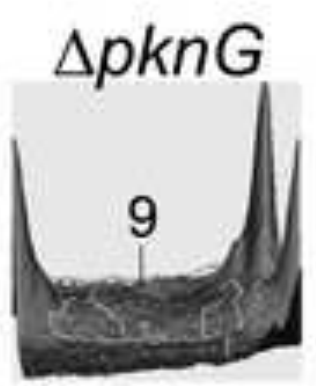

$\Delta p k n G+P k n G$

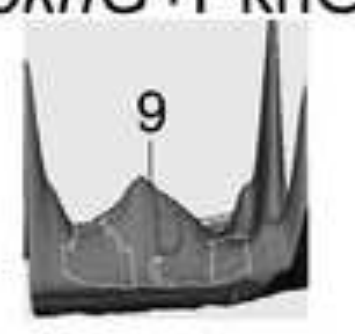


A SVFDDGLif

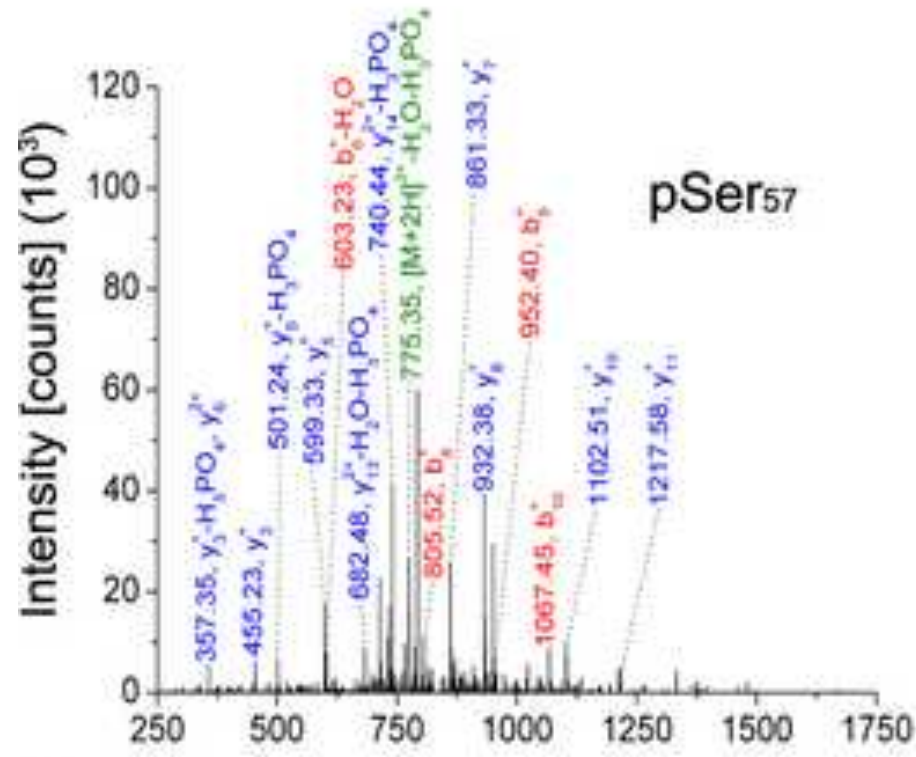

B

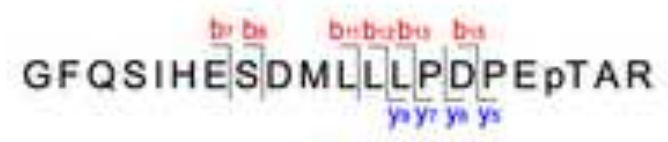

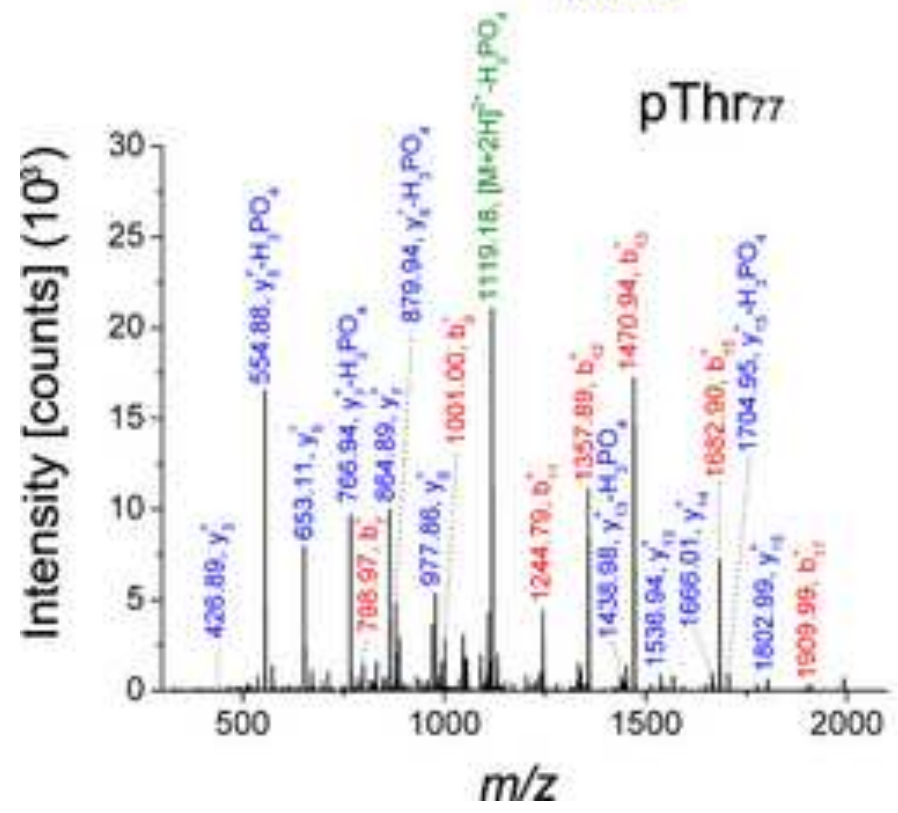

C DLYELPPEEAASIPQTP DTQ DI TSDVIDR

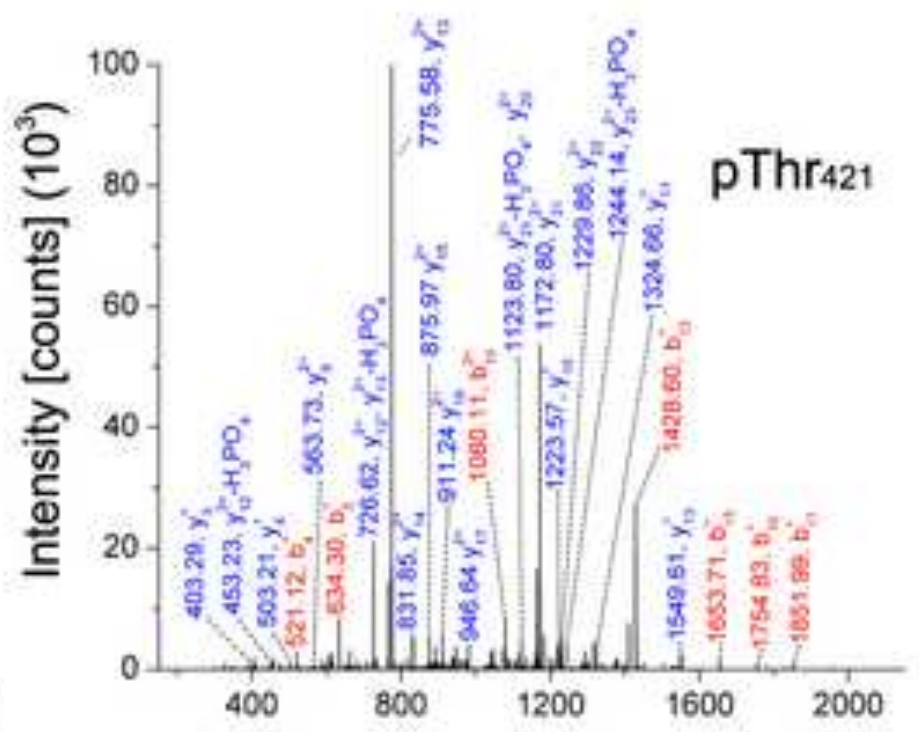

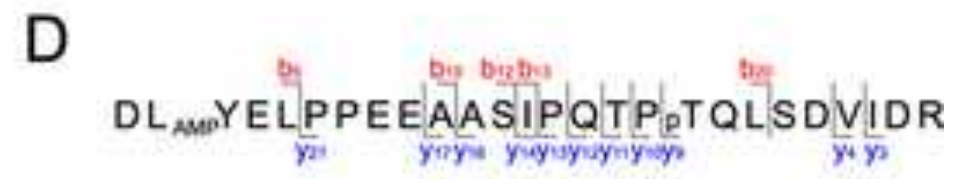

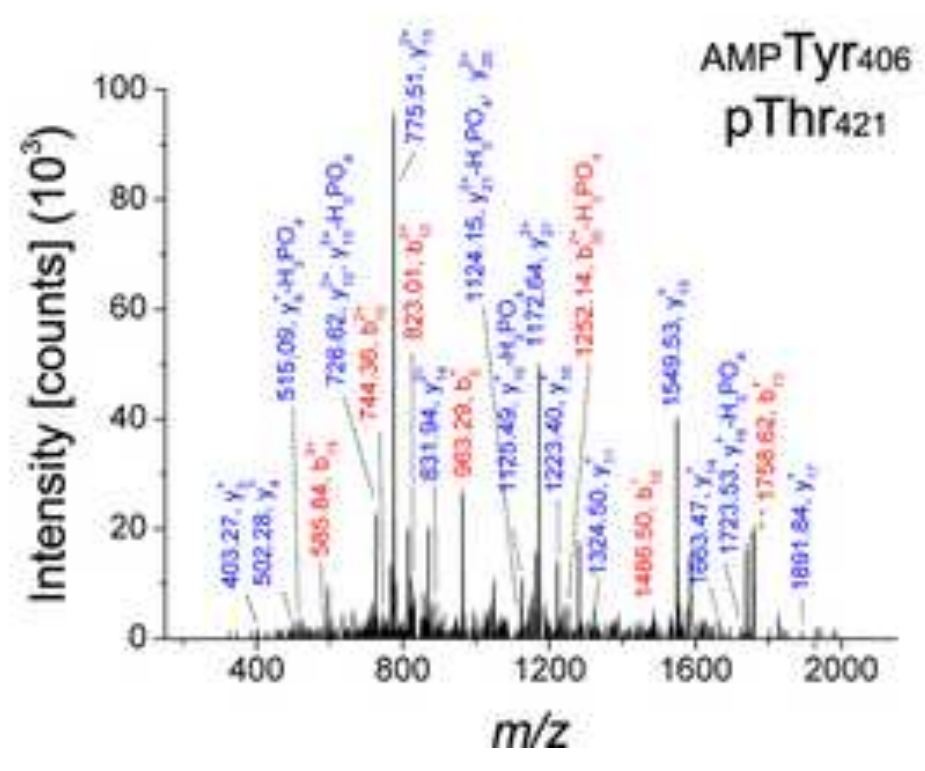



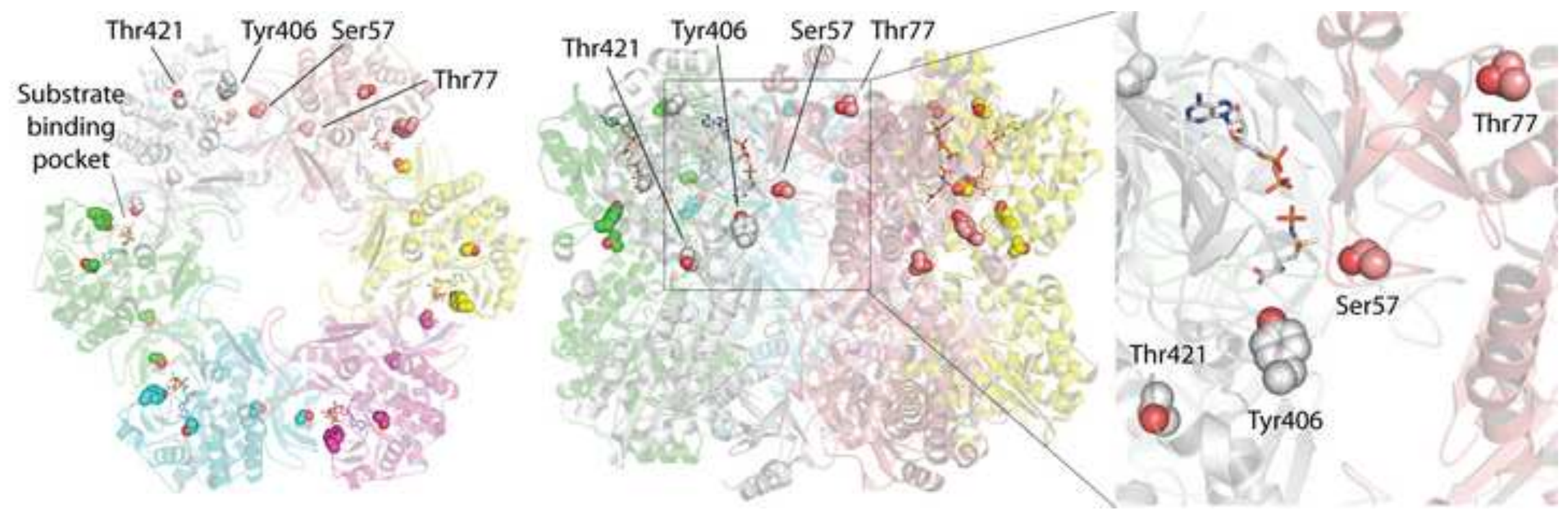

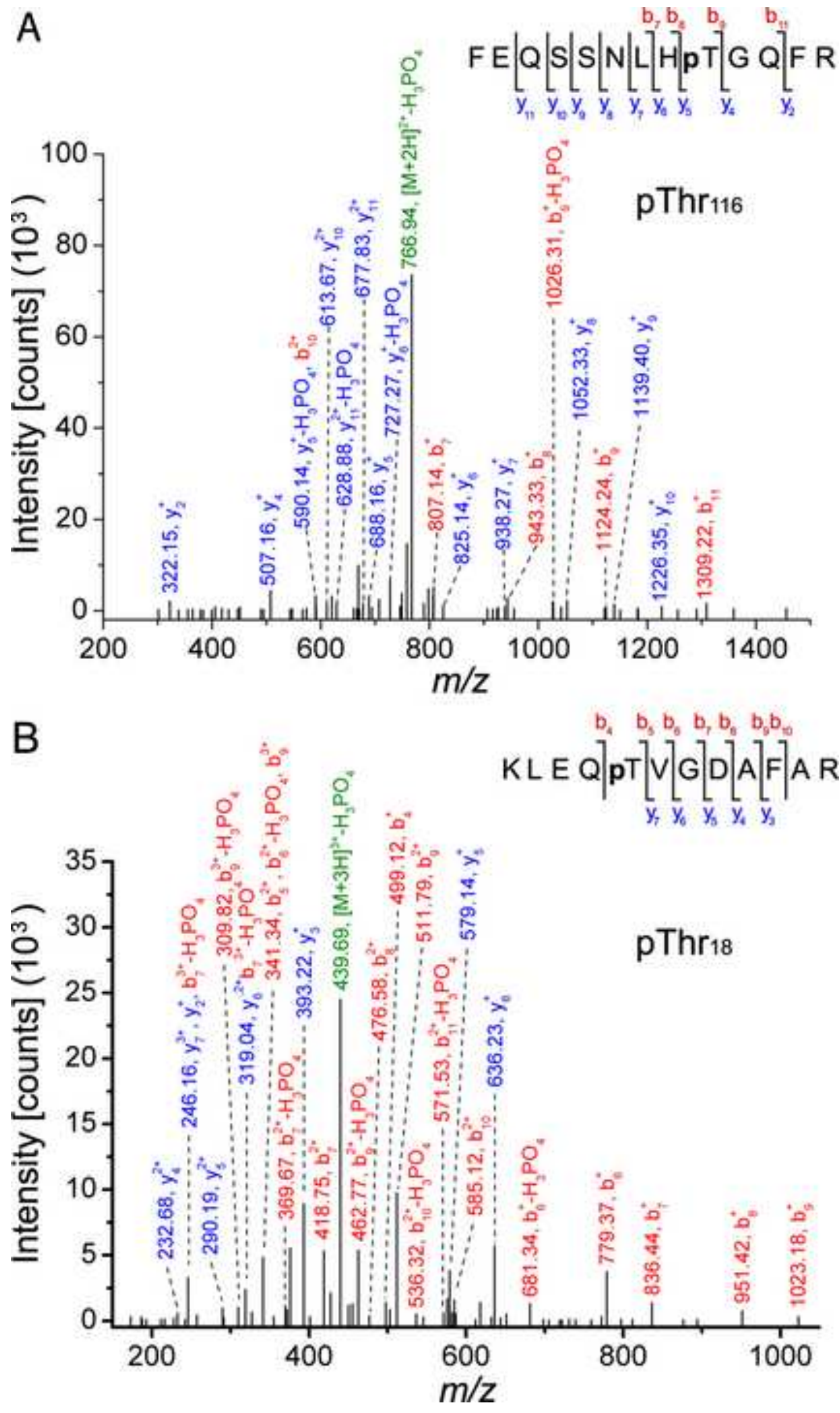
Figure 6

Click here to download high resolution image
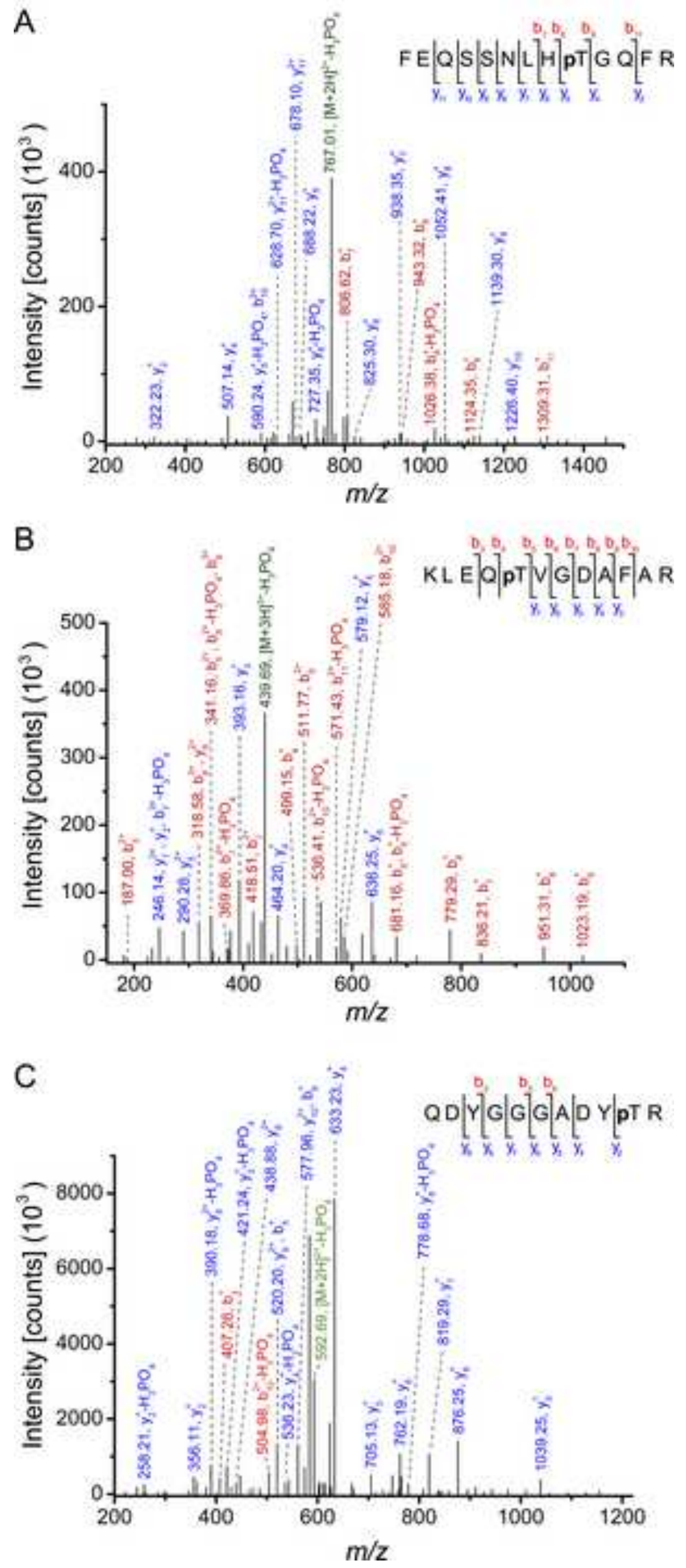